\title{
Cytoglobin Deficiency Promotes Liver Cancer Development from Hepatosteatosis through Activation of the Oxidative Stress Pathway
}

Le Thi Thanh Thuy, ${ }^{*}$ Yoshinari Matsumoto, ${ }^{* \dagger}$ Tuong Thi Van Thuy, ${ }^{*}$ Hoang Hai, ${ }^{*}$ Maito Suoh, ${ }^{\ddagger}$ Yuka Urahara, ${ }^{*}$ Hiroyuki Motoyama, ${ }^{*}$ Hideki Fujii, ${ }^{*}$ Akihiro Tamori, ${ }^{*}$ Shoji Kubo, ${ }^{\$}$ Shigekazu Takemura, ${ }^{\S}$ Takashi Morita, Katsutoshi Yoshizato, ${ }^{*}$ and Norifumi Kawada*

From the Departments of Hepatology, * Medical Education and General Practice, ${ }^{\ddagger}$ Hepato-Biliary-Pancreatic Surgery, ${ }^{\S}$ and Molecular Genetics, ${ }^{\llbracket}$ Graduate School of Medicine, and the Department of Medical Nutrition, ${ }^{\dagger}$ Graduate School of Human Life Science, Osaka City University, Osaka; and the PhoenixBio Co. Ltd., "Hiroshima, Japan

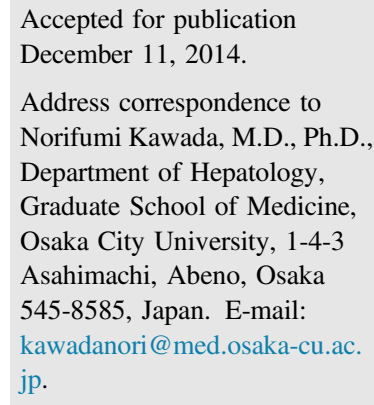

Nonalcoholic steatohepatitis (NASH), an increasingly recognized obesity-related liver disease, is characterized by hepatocyte steatosis accompanied by a fibroinflammatory reaction. ${ }^{1,2}$ Several studies have shown that NASH patients are at risk for progression to cirrhosis, the most common risk factor for hepatocellular carcinoma (HCC). ${ }^{1,3}$ Compared to what is known about the pathogenesis of hepatitis virusinduced $\mathrm{HCC}$, insight into NASH-associated HCC remains immature.

Currently, it is thought that the liver develops NASH via several pathological steps. Hepatocytes undergo degeneration characterized by the accumulation of fatty acids, which are excessively oxidized in the cellular organelles, including mitochondria. During this process, reactive oxygen species (ROS) are produced and trigger oxidative stress, leading to cell and tissue damage. ${ }^{1}$ Hepatic macrophages consisting of resident Kupffer cells and infiltrating bone marrow-derived macrophages produce inflammatory mediators, such as tumor necrosis factor $\alpha$ (TNF- $\alpha$ ), IL-6, IL-1 $\beta$, and ROS. ${ }^{4,5}$ These mediators further stimulate hepatocyte steatosis and initiate the activation of hepatic stellate cells (HSCs). Finally, the persistent secretion of ROS and mediators from these cells induces the development of advanced fibrosis.

\footnotetext{
Supported by Japan Society for the Promotion of Science (JSPS) Grantin-Aid for Young Scientific Research grant 25860554 (L.T.T.T.); JSPS Grant-in-Aid for Scientific Research grants 21390232 (N.K.), 23112518 (N.K.), and 25293177 (N.K.); and Research on Hepatitis and BSE, the Ministry of Health Labor and Welfare (N.K.).

Disclosures: None declared.
} 
Cytoglobin (Cygb) was originally discovered in rat HSCs in $2001,{ }^{6}$ and is the fourth globin to be discovered in mammals. ${ }^{7,8}$ CYGB is present in fibroblasts that store vitamin $\mathrm{A}$ in the visceral organs, including the liver and pancreas. ${ }^{9}$ CYGB facilitates oxygen $\left(\mathrm{O}_{2}\right)$ diffusion through tissues, scavenges nitric oxide (NO) and other ROS, has a protective function during oxidative stress, ${ }^{10}$ and suppresses tumorigenesis. ${ }^{11-14} \mathrm{We}$ previously showed that $C y g b$-deficient $\left(\mathrm{Cygb}^{-1-}\right)$ mice exhibit susceptibility to cancer development in the liver and lung with diethylnitrosamine administration. ${ }^{15}$ Therefore, the absence of CYGB likely promotes a carcinogenic process in the presence of liver disease.

The present study clarifies the role of Cygb in steatohepatitis induced by a choline-deficient amino aciddefined diet (CDAA) in mice. The CDAA diet is a useful model to investigate NASH because it induces fibrosis, systemic insulin resistance, and steatohepatitis, which are compatible to the pathophysiology of human NASH. The administration of CDAA to C57BL/6 wild-type (WT) mice was reported to induce defined liver fibrosis not earlier than 22 weeks, and $\mathrm{HCC}$ nodules at a late time point, 84 weeks. ${ }^{16}$ Herein, we showed $C y g b^{-1-}$ mice fed a CDAA diet, leading to a severe NASH condition and a $100 \%$ incidence of $\mathrm{HCC}$ at an early time point, 32 weeks. Moreover, primary untreated HSCs isolated from $\mathrm{Cygh}^{-/-}$ mice showed a preactivated condition characterized by augmented ROS and cytokine production.

\section{Materials and Methods}

\section{Human Tissues and Specimens}

Human NASH specimens $(n=15)$, used for immunohistochemistry (IHC) of CYGB, were obtained from patients in Osaka City University Hospital (Osaka, Japan), who were diagnosed with NASH according to the classification of Matteoni et al. ${ }^{17}$ Intact human specimens $(n=3)$ of nontumor lesions were obtained from patients who had metastasis liver tumors or cholangiocarcinoma treated by surgical resection. HCC tissues and noncancerous liver tissues were obtained from nine patients without hepatitis virus B or C infection, who had undergone a hepatectomy at the Osaka City University Hospital. They were patients with almost intact liver $(n=2)$, fatty liver $(n=1)$, liver fibrosis by undetermined etiology $(n=1)$, NASH $(n=1)$, and alcoholism $(n=4)$. The specimens were routinely processed, formalin fixed, and paraffin embedded. A portion of tissues was frozen and stored at $-80^{\circ} \mathrm{C}$ without fixation. RNAs were extracted from them by the acid guanidinium thiocyanate-phenol-chloroform method, as described in our previous study. ${ }^{18}$ All patients gave written informed consent to participate in this study in accordance with the ethical guidelines of the 1975 Declaration of Helsinki, and according to the process approved by the ethical committee of Osaka City University, Graduate School of Medicine.
Mice and Diet

C57BL/6 Cygb conventional knockout mice were generated in our laboratory, as described previously. ${ }^{15} \mathrm{C} 57 \mathrm{BL} / 6$ mice (WT) were purchased from SLC (Shizuoka, Japan).

For the NASH model, $78 \mathrm{Cygb}^{-/-}$and 77 WT mice were used, including males and females. Eight-week-old mice were fed CDAA (catalog 518753; Dyets, Bethlehem, PA) or a control diet, choline-supplied amino acid-defined diet (CSAA; catalog 518754; Dyets) with $n=5$ to 14 per group. The CSAA control diet induces simple steatosis, but neither inflammation nor fibrosis, in WT mice ${ }^{16}$ (Supplemental Figure S1). Mice were fed these diets continuously for 8 , 16 , or 32 weeks. To investigate tissue hypoxia, 1 hour before sacrifice, some mice were injected i.p. with hydroxyprobe- 1 solution at a dose of $60 \mathrm{mg} / \mathrm{kg}$ body weight using the hydroxyprobe-1 Omni Kit (Hydroxyprobe, Burlington, MA), according to the manufacturer's protocol.

In the macrophage-depletion experiment, a subgroup of 20 mice were divided into four groups. A short 8-week protocol on the CDAA diet followed, with macrophage deletion in the final week, which was used to examine the early events of NASH. At the seventh week of CDAA feeding, Kupffer cell depletion was induced by injecting $200 \mu \mathrm{L}$ liposomal clodronate (FormuMax Scientific, Palo Alto, CA) into the mouse tail vein, according to the manufacturer's protocol. Control mice were injected with the same amount of plain control liposomes. Mice were continuously fed the CDAA diet and sacrificed 1 week after injection.

For N-acetyl cysteine (NAC) treatment, a total of 53 Cyg $b^{-1-}$ and WT mice, divided into six groups $(n=5$ to 13 per group), were fed the CDAA diet, together with 0.1 $\mathrm{mmol} / \mathrm{L}$ NAC (Sigma-Aldrich, St. Louis, MO) in the drinking water for 2,8 , or 32 weeks, starting at 8 weeks of age. NAC was prepared as a $0.5 \mathrm{~mol} / \mathrm{L}$ stock in sterile water once a month, aliquoted, and stored at $-30^{\circ} \mathrm{C}$ in the dark. Sterile drinking water was freshly made from the stock and changed twice a week. Animal care and procedures were approved by the Osaka City University Animal Care and Use Committee, as set forth in the NIH Guide for the Care and Use of Laboratory Animals. ${ }^{19}$

\section{Histological, IHC, and Immunofluorescence Analysis}

Hematoxylin and eosin, IHC, and immunofluorescence analyses were performed as previously described. ${ }^{15}$ The primary antibodies used for mouse and human samples, including CYGB antibodies, were generated by our laboratory $^{6,15,20}$ and are described in Table 1. Pathological severity of nonalcoholic fatty liver disease was assessed using previously described criteria. ${ }^{21}$ To quantify liver fibrosis, sections ( $5 \mu \mathrm{m}$ thick) were stained with Picrosirius red (Sigma-Aldrich) and counterstained with Fast Green (Sigma-Aldrich). Collagen stained with Sirius Red was quantitated in the sections that were randomly chosen ( $<100$ magnifications, 10 to 20 fields each from sample) 
using Micro Analysis software version 1.1d (Thermo Scientific, West Palm Beach, FL). To quantify CYGB-positive cells in IHC staining, human liver normal $(n=3)$ and NASH sections $(n=5$ in each group of NASH score $\leq 2,3$ to 6 , and 7 to 8 ) were counted in at least 10 high-power fields $(\times 400$ magnification) per section.

\section{DHE Assay}

To examine the oxidative stress condition induced by CDAA diet and by the absence of CYGB, primary HSCs cultured as described below or freshly prepared frozen liver sections, which were warmed up at $37^{\circ} \mathrm{C}$ for 2 hours, were incubated with $2 \mu \mathrm{mol} / \mathrm{L}$ dihydroethidium (DHE; Invitrogen, Eugene, OR) in phosphate-buffered saline for 30 minutes at $37^{\circ} \mathrm{C}$. Then, they were counterstained with DAPI and observed under fluorescent microscopy.

\section{Hydroxyproline Assay}

Hydroxyproline content of the liver was measured by a spectrophotometric assay by using Hydroxyproline Assay Kit (BioVision, Milpitas, CA), according to the assay protocol. Briefly, liver tissue was homogenized in ice-cold distilled water $(100 \mu \mathrm{L}$ of water for every $10 \mathrm{mg}$ of tissue) using a polytron homogenizer. Subsequently, one volume of $12 \mathrm{~N} \mathrm{HCl}$ was added to the homogenized sample in a pressure-tight, Teflon-capped vial and was hydrolyzed for 3 hours at $120^{\circ} \mathrm{C}$. After hydrolysis, $10 \mu \mathrm{L}$ of each hydrolyzed sample was transferred to a 96-well plate and evaporated to dryness under vacuum. Then, samples were oxidized with chloramine-T (Sigma-Aldrich) for 5 minutes at room temperature. The reaction mixture was then incubated in dimethylaminobenzaldehyde at $60^{\circ} \mathrm{C}$ for 90 minutes and cooled to room temperature. A series of wells of hydroxyproline standard were prepared for each assay. Sample absorbance was measured at $560 \mathrm{~nm}$. Hydroxyproline content was expressed as microgram of hydroxyproline per gram liver.

\section{ALT Measurement}

Alanine aminotransferase (ALT) activity (UV test at $37^{\circ} \mathrm{C}$ ) was measured in serum using a commercially available kit (Wako, Osaka, Japan), according to manufacturer's protocol.

\section{Quantitative Real-Time PCR}

Total RNA was extracted from cells and liver tissues using the miRNeasy Mini Kit (Qiagen, Valencia, CA). cDNAs were synthesized using total RNA, a ReverTra Ace qPCR RT Kit (Toyobo, Osaka, Japan) and oligo(dT) ${ }_{12-18}$ primers, according to the manufacturer's instructions. Gene expression was measured by real-time PCR using the cDNAs, SYBR qPCR Mix Reagents (Toyobo), and gene-specific oligonucleotide primers (Table 2) with an ABI Prism 7500 Fast RealTime PCR System (Applied Biosystems, Foster, CA). Glyceraldehyde-3-phosphate dehydrogenase (Gapdh) level was used to normalize the relative abundance of mRNAs.

\section{Gene Expression Profile for Specific Pathway}

The Mouse Oxidative Stress and Antioxidant Defense RT ${ }^{2}$ Profiler PCR Array from SA Biosciences (Frederick, MD; catalog PAMM-065) was performed to examine the expression of 84 genes related to oxidative stress, according

Table 1 Summary of Primary Antibodies Used for Immunohistochemistry or Immunofluorescences

\begin{tabular}{llll}
\hline Antigen & Source & Name/clone; catalog no. & Incubation \\
\hline AFP & US Biological (Swampscott, MA) & F4100-16A (Go) & $0 / \mathrm{N} 4^{\circ} \mathrm{C}, 1: 20$ \\
CD68 & Abcam (Cambridge, UK) & Polyclonal (Rb); ab125212 & $0 / \mathrm{N} 4^{\circ} \mathrm{C}, 1: 300$ \\
53PB1 & Abcam & Polyclonal (Rb); ab36823 & $0 / \mathrm{N} 4^{\circ} \mathrm{C}, 1: 300$ \\
CYGB & Our laboratory & Polyclonal (Rb) anti-mouse & $0 / \mathrm{N} 4^{\circ} \mathrm{C}, 1: 100$ \\
CYGB & Our laboratory & Monoclonal (Rb) anti-human & $0 / \mathrm{N} 4^{\circ} \mathrm{C}, 1: 100$ \\
p-AKT & Cell Signaling (Danvers, MA) & Monoclonal (Rb); 3787 & $0 / \mathrm{N} 4^{\circ} \mathrm{C}, 1: 300$ \\
F4/80 & eBioscience (San Diego, CA) & Monoclonal (Rt); 14-4801 & $0 / \mathrm{N} 4^{\circ} \mathrm{C}, 1: 200$ \\
H0-1 & Assay designs (Ann Arbor, MI) & Polyclonal (Rb); SPA-895 & 30 minutes room temperature, $1: 100$ \\
Hydroxyprobe-1 & Hydroxyprobe, Inc. (Burlington, MA) & Rb anti-pimonidazole; PAb2627 & $0 / \mathrm{N} 4^{\circ} \mathrm{C}, 1: 100$ \\
iN0S & Abcam & Polyclonal (Rb); ab15203 & $0 / \mathrm{N} 4^{\circ} \mathrm{C}, 1: 100$ \\
Ki-67 & Abcam & Monoclonal (Rb); ab16667 & $0 / \mathrm{N} 4^{\circ} \mathrm{C}, 1: 100$ \\
MP0 & Abcam & Polyclonal (Rb); ab45977 & $0 / \mathrm{N} 4^{\circ} \mathrm{C}, 1: 300$ \\
Neutrophil & Abcam & Monoclonal (Rt); ab2557 & $0 / \mathrm{N} 4^{\circ} \mathrm{C}, 1: 100$ \\
Nitrotyrosine & Cell Signaling & Polyclonal (Rb); 9691 & $0 / \mathrm{N} 4^{\circ} \mathrm{C}, 1: 100$ \\
p-ERK & Cell Signaling & Monoclonal (Rb); 4370 & $0 / \mathrm{N} 4^{\circ} \mathrm{C}, 1: 200$ \\
aSma & Sigma-Aldrich & Monoclonal (Mo); clone: $1 \mathrm{~A} 4$ & $0 / \mathrm{N} 4^{\circ} \mathrm{C}, 1: 300$ \\
rH2AX & Novus Biologicals (Littleton, CO) & Monoclonal (Rb); NB100-79967 & $0 / \mathrm{N} 4^{\circ} \mathrm{C}, 1: 200$ \\
\hline
\end{tabular}

${ }^{*}$ All antigens were retrieved by autoclaving for 15 minutes in $0.01 \mathrm{~mol} / \mathrm{L}$ citrate buffer containing $0.05 \%$ Tween 20 (pH 6.0), except for Neutrophile and $\mathrm{F} 4 / 80$, in which proteinase $\mathrm{K}(400 \mu \mathrm{g} / \mathrm{mL})$ in TE buffer $(\mathrm{pH} 8.0)$ was used.

AFP, $\alpha$-fetoprotein; CYGB, cytoglobin; ERK, extracellular signal-regulated kinase; Go, goat; HO, heme oxygenase; H2AX, phosphorylated H2A histone protein, member $\mathrm{X}$; iNOS, inducible nitric oxide synthase; Mo, mouse; MPO, myeloperoxidase; 0/N, overnight; Rb, rabbit; Rt, rat; Sma, smooth muscle actin. 
Table 2 Human and Mouse Primers Used for Quantitative RealTime PCR

\begin{tabular}{|c|c|}
\hline $\begin{array}{l}\text { Primer } \\
\text { name/ } \\
\text { gene* }^{*}\end{array}$ & Sequence \\
\hline \multirow[t]{2}{*}{$h C Y G B$} & F: 5'-TGCCAGTGACTTCCCACCT-3' \\
\hline & R: 5'-TAGATGAGGCCACGCAGC-3' \\
\hline \multirow[t]{2}{*}{$h G A P D H$} & F: 5'-GCACCGTCAAGGCTGAGAAC-3' \\
\hline & R: 5'-TGGTGAAGACGCCAGTGGA-3' \\
\hline \multirow{2}{*}{$m A f p$} & F: 5'-CACACCCGCTTCCCTCAT-3' \\
\hline & R: 5'-TTTTCGTGCAATGCTTTGGA-3' \\
\hline \multirow[t]{2}{*}{$m B c l 2$} & F: 5'-AAGGGCTTCACACCCAAATCT-3' \\
\hline & R: 5'-CTTCTACGTCTGCTTGGCTTTGA-3' \\
\hline \multirow[t]{2}{*}{$m C a t$} & F: 5'-ATGGCTTTTGACCCAAGCAA-3' \\
\hline & R: 5'-CGGCCCTGAAGCTTTTTGT-3' \\
\hline \multirow[t]{2}{*}{$m C c l 3$} & F: 5'-TGAAACCAGCAGCCTTTGCTC-3' \\
\hline & R: 5'-AGGCATTCAGTTCCAGGTCAGTG-3' \\
\hline \multirow[t]{2}{*}{$m C c l 4$} & F: 5'-CCATGAAGCTCTGCGTGTCTG-3' \\
\hline & R: 5'-GGCTTGGAGCAAAGACTGCTG-3' \\
\hline \multirow[t]{2}{*}{ mFos } & F: 5'-CCCCAAACTTCGACCATGAT-3' \\
\hline & R: 5'-GGAGGATGACGCCTCGTAGTC-3' \\
\hline \multirow[t]{2}{*}{ mJun } & F: 5'-CCGCCCCTGTCCCCTAT-3' \\
\hline & R: 5'-TCCTCATGCGCTTCCTCTCT-3' \\
\hline \multirow[t]{2}{*}{ mCol1a1 } & F: $5^{\prime}-\mathrm{CCTCCCGCACCCAGTTC-3^{ \prime }}$ \\
\hline & R: 5'-CATCAGCATGTTTGGAGTAGTAAGC-3' \\
\hline \multirow[t]{2}{*}{$m C x c l 1$} & F: 5'-TGAGCTGCGCTGTCAGTGCCT-3' \\
\hline & R: 5'-AGAAGCCAGCGTTCACCAGA-3' \\
\hline \multirow[t]{2}{*}{$m C x c l 2$} & F: 5'-GAGCTTGAGTGTGACGCCCCCAGG-3' \\
\hline & R: 5'-GTTAGCCTTGCCTTTGTTCAGTATC-3' \\
\hline \multirow[t]{2}{*}{$m C x c l 5$} & F: 5'-GCATTTCTGTTGCTGTTCACGCTG-3' \\
\hline & R: 5'-CCTCCTTCTGGTTTTTCAGTTTAGC-3' \\
\hline \multirow[t]{2}{*}{$m C x c l 7$} & F: 5'-TGGGCCTGATCCTTGTTGCGC-3' \\
\hline & R: 5'-GCACCGTTTTTTGTCCATTCTTCAG-3' \\
\hline \multirow[t]{2}{*}{$m C c n d 1$} & F: 5'-GCCCGGAGGGATTTGC-3' \\
\hline & R: 5'-AGACGGAACACTAGAACCTAACAGATT-3' \\
\hline \multirow[t]{2}{*}{$m C y g b$} & F: 5'-TGCATGACCCAGACAAGGTA-3' \\
\hline & R: 5'-GGTCACGTGGCTGTAGATGA-3' \\
\hline \multirow[t]{2}{*}{ mGapdh } & F: 5'-TGCACCACCAACTGCTTAG- $3^{\prime}$ \\
\hline & R: 5'-GGATGCAGGGATGATGTTC-3' \\
\hline \multirow[t]{2}{*}{$m G p \times 6$} & F: 5'-GCCCAGAAGTTGTGGGGTTC-3' \\
\hline & R: 5'-TCCATACTCATAGACGGTGCC-3' \\
\hline \multirow[t]{2}{*}{$m H o-1$} & F: 5'-GGTGATGGCTTCCTTGTACC-3' \\
\hline & R: 5'-AGTGAGGCCCATACCAGAAG- $3^{\prime}$ \\
\hline \multirow[t]{2}{*}{ mHif1a } & F: 5'-CAGTACAGGATGCTTGCCAAAA-3' \\
\hline & R: 5'-ATACCACTTACAACATAATTCACACACACA-3' \\
\hline \multirow[t]{2}{*}{ mIl1b } & F: 5'-CCATGGCACATTCTGTTCAAA-3' \\
\hline & R: 5'-GCCCATCAGAGGCAAGGA-3' \\
\hline mIl6 & F: 5'-CCGCTATGAAGTTCCTCTCTGC-3' \\
\hline & R: $5^{\prime}$-АTCCTCTGTGAAGTCTCCTCTCC-3' \\
\hline m-iNos & F: 5'-CCTGGTACGGGCATTGCT-3' \\
\hline & R: 5'-GCTCATGCGGCCTCCTTT-3' \\
\hline$m C c l 2$ & F: 5'-GAGAGCCAGACGGGAGGAAG-3' \\
\hline & R: 5'-TGAATGAGTAGCAGCAGGTGAG-3' \\
\hline mMpo & F: 5'-CCATGGTCCAGATCATCACA-3' \\
\hline & R: 5'-GCCGGTACTGATTGTTCAGG-3' \\
\hline$m T g f b 1$ & F: 5'-GAGCCCGAAGCGGACTACT-3' \\
\hline & R: 5'-TTGCGGTCCACCATTAGCA-3' \\
\hline$m T g f b 3$ & F: 5'-AGGGCCCTGGACACCAATTAC-3' \\
\hline & R: 5'-CCTTAGGTTCTGGGACCCATTTC-3' \\
\hline
\end{tabular}

(table continues)
Table 2 (continued)

\begin{tabular}{ll}
\hline $\begin{array}{l}\text { Primer } \\
\text { name/ } \\
\text { gene* }\end{array}$ & Sequence \\
\hline mTimp1 & F: 5'-ACTCGGACCTGGTCATAAGGGC-3' \\
& R: 5'-TTCCGTGGCAGGCAAGCAAAGT-3' \\
mTnfa & F: 5'-CTCTTCTCATTCCTGCTTGTGG-3' \\
& R: 5'-AATCGGCTGACGGTGTGG-3' \\
m- $\alpha$ Sma & F: 5'-TCCCTGGAGAAGAGCTACGAACT-3' \\
& R: 5'-AAGCGTTCGTTTCCAATGGT-3' \\
\hline
\end{tabular}

*h, human; $m$, mouse.

CYGB, cytoglobin; F, forward; GAPDH, glyceraldehyde-3-phosphate dehydrogenase; iNos, inducible nitric oxide synthase; $R$, reverse; Sma, smooth muscle actin.

to manufacturer's protocol. Briefly, $1 \mu \mathrm{g}$ of total RNA from 16-week-old CDAA-fed WT or $C y g b^{-l-}$ mice was used to make first-strand cDNA using $\mathrm{RT}^{2}$ First Strand Kit (SA Biosciences). PCR mixture containing cDNA, distilled water, and SYBR Green master mix (SA Biosciences) was loaded into each well of 96-well plates containing the predispensed gene-specific primer sets, and PCR was performed with an ABI Prism 7500 Fast Real-Time PCR System (Applied Biosystems). The PCR was performed in 96-well plates with 84 genes related to oxidative stress, five housekeeping genes (Actb, Gapdh, Hsp90abl, Hprtl, and Gusb) used for normalizing the PCR array data, one negative control to verify genomic DNA contamination, and three wells of RT controls to verify the efficiency of the RT reaction. The excel-based PCR array data analysis (SA Biosciences) was used to calculate the $\mathrm{C}_{\mathrm{T}}$ values for all of the genes in the array. Then, fold changes in gene expression for pairwise comparison using the $\Delta \Delta \mathrm{C}_{\mathrm{T}}$ method were used to determine the relative expression levels of genes of interest for each sample.

\section{Immunoblot Analysis}

Protein samples (10 to $40 \mu \mathrm{g}$ ) were subjected to SDS-PAGE and transferred to Immobilon P membranes (Millipore Corp., Bedford, MA). After blocking, membranes were probed with primary antibodies against CYGB (1: 500) from our laboratory (Table 1), AKT (1:1000; Cell Signaling, Danvers, MA), phosphorylated AKT (1:500; Cell Signaling), BCL-2 (1:1000; Cell Signaling), extracellular signal-regulated kinase (ERK; 1:500; Cell Signaling), phosphorylated ERK (1:1000; Cell Signaling), CYCLIN D1 (1:5000; Cell Signaling), phosphorylated SMAD3 (1:1000; Abcam, Cambridge, UK), total SMAD3 (1:1000; Abcam), heme oxygenase-1 (HO-1; 1:1000; Cosmo Bio Co Ltd, Tokyo, Japan), myeloperoxidase (1:1000; Abcam), $\alpha$-smooth muscle actin ( $\alpha$-Sma; 1:1000; Abcam), or GAPDH (1:2000; Santa Cruz Biotechnology, Santa Cruz, CA). Membranes were then incubated with horseradish peroxidase-conjugated secondary antibodies at 1:2000 dilutions. Immunoreactive bands 
were visualized using the electrochemiluminescence detecting reagent (GE Healthcare UK Ltd, Buckinghamshire, UK), and documented with the Fujifilm Image Reader LAS-3000 (Fujifilm, Tokyo, Japan) coupled with image analysis software (Multi Gauge version 3.1; Fujifilm).

\section{Cells}

HSCs were isolated from WT (HSC Cygb-wild $)$ and $\mathrm{Cyg}^{-1-}$ $\left(\mathrm{HSC}^{\text {Cygb-null}}\right)$ mice using the pronase-collagenase digestion method, as previously described, ${ }^{22}$ and were cultured on uncoated plastic dishes (BD Falcon, Franklin Lake, NY) or glass chamber slides (Thermo Fisher Scientific, Waltham, MA) in Dulbecco's modified Eagle's medium (SigmaAldrich) supplemented with $10 \%$ fetal bovine serum (Invitrogen, Carlsbad, CA) and antibiotics $(100 \mathrm{U} / \mathrm{mL}$ penicillin and $100 \mu \mathrm{g} / \mathrm{mL}$ streptomycin). $\mathrm{HSC}^{\text {Cygb-wild }}$ and $\mathrm{HSC}^{\text {Cygb-null }}$ cells were harvested at days 1, 4, and 7 for RNA, protein extractions, or for immunofluorescence, Oil Red O staining.

\section{siRNA Transient Transfection}

siRNA $C y g b$ or the siRNA negative control (Ambion, Austin, TX) was transfected into HSC ${ }^{\text {Cygb-wild }}$ using Lipofectamine RNAiMAX Transfection Reagent (Invitrogen, Carlsbad, CA) at a final concentration of $50 \mathrm{nmol} / \mathrm{L}$, as previously described. ${ }^{23}$ After 24 hours, the culture medium was changed to fresh Dulbecco's modified Eagle's medium (Sigma-Aldrich) supplemented with 10\% fetal bovine serum (Invitrogen) and antibiotic. Then, after 72 hours, the cells were collected for total RNA extraction or after 96 hours, they were collected for protein extraction and for double immunofluorescence of $\alpha$-SMA and HO- 1 .

\section{Recombinant Human $C Y G B$ Treatment}

Primary $\mathrm{HSC}^{\text {Cygb-null }}$ mice were isolated from $\mathrm{Cygh}^{-/-}$ mice and cultured on uncoated plastic dishes. After 24 hours, the culture medium was supplemented with $100 \mu \mathrm{g} /$ $\mathrm{mL}$ of recombinant human $C Y G B .{ }^{10}$ And, after 72 hours, the cells were subjected for mRNA and protein analysis of $\alpha$ SMA and CYGB expression.

\section{Statistical Analysis}

All data are expressed as the means \pm SEM. Two groups were compared using an unpaired Student's $t$-test (twotailed). $P<0.05$ was considered statistically significant.

\section{Results}

\section{Expression of CYGB in Human NASH and HCC}

CYGB was originally identified in rat $\mathrm{HSCs}^{6}$; however, its expression in human NASH livers has remained undetermined. In normal human liver, CYGB was expressed in cells in Disse's space that contained lipid droplets and were negative for $\alpha$-SMA, but not in hepatocytes (Figure 1A), indicating that CYGB-positive cells are HSCs. In NASH livers, the expression of CYGB declined in a negative correlation with increased NASH score (Figure 1B). A similar decline in CYGB protein (Figure 1C) and mRNA (Figure 1D) expression was observed in HCC regions. Therefore, a decline of CYGB expression likely contributes to the development of human NASH and liver cancer.
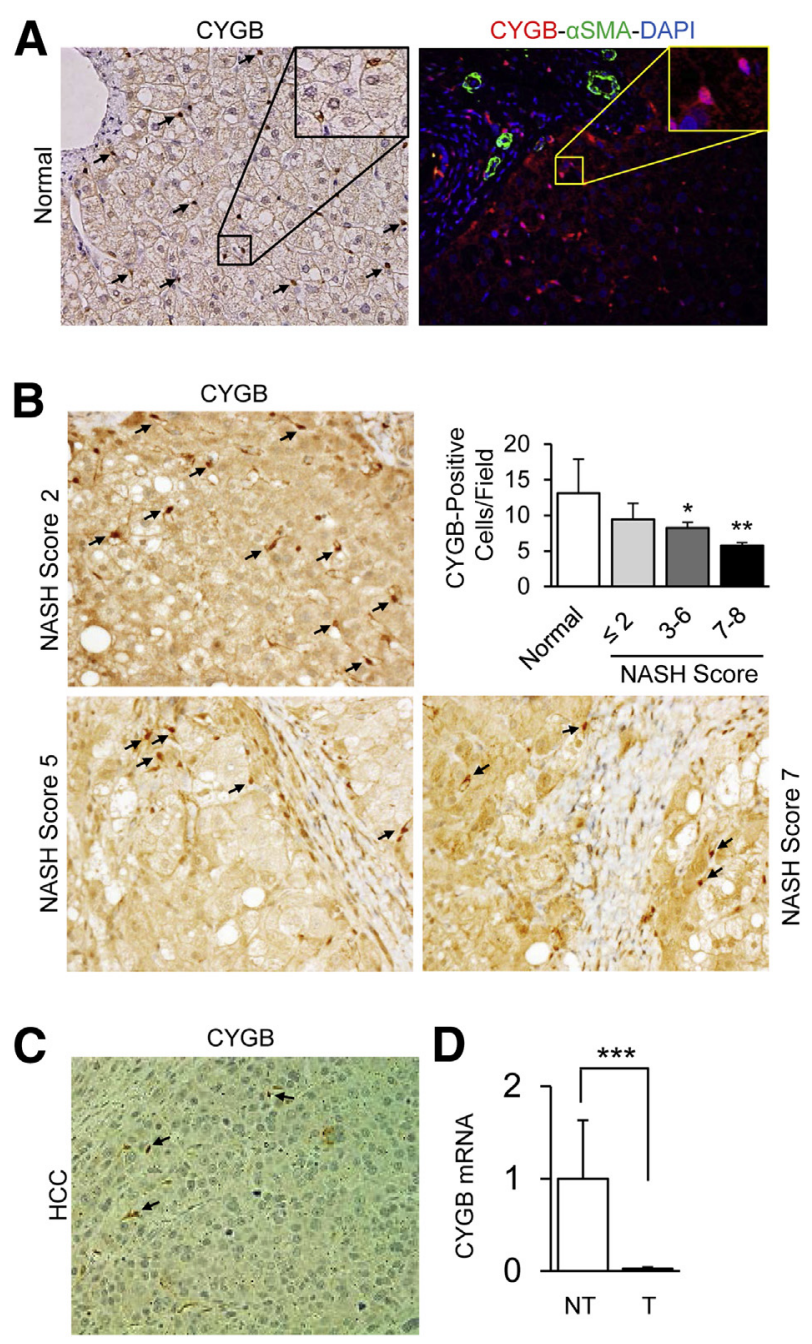

Figure 1 Expression of cytoglobin (CYGB) in human liver. A: Immunohistochemistry of CYGB and immunofluorescence of CYGB and $\alpha$ smooth muscle actin ( $\alpha$-SMA) in normal human liver. B: Immunohistochemistry of CYGB and the quantification of its expression in human nonalcoholic steatohepatitis (NASH) livers with NASH score from 2 to 8. C and D: CYGB expression at the protein (C) and mRNA (D) levels in hepatocellular carcinoma [HCC; tumor (T)] and nontumor (NT) tissues derived from $\mathrm{HCC}$ patients without hepatitis $\mathrm{B}$ or $\mathrm{C}$ virus infection. Arrows indicate hepatic stellate cells. Data represent the means \pm SD. $n=3(\mathbf{A}) ; n=5$ in each group (B); $n=9$ (C and D). ${ }^{*} P<0.05$, ${ }^{* * P}<0.01$, and ${ }^{* * * P}<0.001$. Original magnifications: $\times 400(\mathbf{A}-\mathbf{C})$; $\times 800$ (insets, A). 
Aggravation of Steatohepatitis and Liver Fibrosis in Cygb Deficiency

On the basis of observations in humans, we investigated Cygb involvement in the pathogenesis of NASH using $C_{y g} b^{-1-}$ and WT mice fed a CDAA or control CSAA diet. The control diet induced simple steatosis in both sexes of WT mice, as shown by microscopy and hematoxylin and eosin staining (Supplemental Figure S1, A and B). WT mice fed the CDAA diet exhibited time-dependent hepatomegaly, as indicated by the increased liver per body weight ratio in both males and females. However, these ratios were significantly lower in $\mathrm{Cygb}^{-/-}$mice; those livers exhibited atrophy and surface irregularity, indicating liver fibrosis development (Supplemental Figure S1).

At as early as 8 weeks of CDAA treatment, the WT liver showed minor steatosis and almost no fibrosis development, whereas the $\mathrm{Cygb}^{-1-}$ mouse liver exhibited inflammatory cell accumulation, including F4/80-positive macrophages, collagen deposition, especially along hepatic sinusoids, and prominent steatosis, as demonstrated by hematoxylin and eosin, Sirius Red, and Oil red O staining, respectively (Figure 2A). Concomitantly, the hepatocyte damage was more severe in $\mathrm{Cygb}^{-1-}$ mice compared to WT mice, as indicated by the higher serum ALT level (Figure 2B). All of these changes were more obvious at 16 weeks and most severe at 32 weeks of CDAA feeding, as assessed by the Sirius Red-positive area, hydroxyproline content, and total NASH score (Figure 2, A-C). The absence of CYGB in HSCs (Supplemental Figure S2) induced markedly increased $\alpha$-Sma expression, which clearly revealed the activation of HSCs from an early stage (Figure 2A), together with increased mRNA levels of $\alpha$-Sma, collagen 1a1 (Figure 2D), tissue inhibitor of metalloproteinase 1, and transforming growth factor- $\beta$ (data not shown) in the livers of $C y g b^{-l-}$ mice. Subsequently, phosphorylation of SMAD3, a key protein involved in the transforming growth factor- $\beta$-dependent fibrotic pathway, was up-regulated in $\mathrm{Cygb}^{--1}$ mice, indicative of the activation of a fibrotic signal in the early stage of CDAA diet feeding (Figure 2E). These results demonstrate that the absence of CYGB accelerates all aspects of the pathological processes of CDAAinduced steatohepatitis in mice.

\section{Cygb Deficiency-Induced Inflammation and Liver Cancer Development}

After 32 weeks of CDAA treatment, liver tumors developed in $100 \%$ of both male and female $\mathrm{Cygb}^{-1-}$ mice, but never in their WT counterparts (Figure 2A and Supplemental Figure S1). The average number of nodules per mouse and the size of the nodules in male $\mathrm{Cygh}^{-1-}$ mice were $4.20 \pm 3.39$ $\mathrm{mm}$ and $3.81 \pm 2.91 \mathrm{~mm}$, respectively, which was smaller in female $\mathrm{Cygb}^{-1-}$ mice (Supplemental Figure S1). The liver tumor induction in female $\mathrm{Cygb}^{-1-}$ mice is surprising because WT female mice are usually resistant to tumor formation. ${ }^{24,25}$
The tumors in $\mathrm{Cyg}^{-1-}$ livers had increased $\alpha$-fetoprotein expression, Ki-67-positive nuclei, and ERK phosphorylation (Figure 3A). Expression of 53BP-1 and $\gamma \mathrm{H} 2 \mathrm{AX}$, indicators of DNA double strain break, was markedly elevated in both tumor and nontumor regions of CDAAfed $\mathrm{Cygb}^{-1-}$ mouse livers, but were negligible in the WT mice (Figure 3A). Assuming that the DNA damage precedes the development of liver tumor, we assessed the $\gamma \mathrm{H} 2 \mathrm{AX}$ expression at earlier time points (8 or 16 weeks) on the CDAA and CSAA diet (Supplemental Figure S3A). $\gamma \mathrm{H} 2 \mathrm{AX}$ was negative in all CSAA groups, in both WT and $\mathrm{Cygb}^{-1-}$ mice. In CDAA-treated mice, there were some $\gamma \mathrm{H} 2 \mathrm{AX}$-positive hepatocytes in $\mathrm{Cygb}^{-1-}$ mouse livers, but not in WT ones, at 8 or 16 weeks. In addition, mRNA expression for Afp, cytokines, such as Il-6, Il-1 $\beta$, Tnf- $\alpha$, and transforming growth factor- $\beta 1$, and chemokines, such as Cxcl 2 and Ccls 2 to 4, was significantly increased in $\mathrm{Cygh}^{-/-}$ mice (Figure 3B). Their downstream targets, ERK, AKT, CYCLIN D1, and BCL-2, at the protein level, and cJun, cFos, Cyclin D1, and Bcl-2, at the mRNA level, were induced and activated in $\mathrm{Cygh}^{-1-}$ mice fed a CDAA diet (Figure 3, C and $\mathrm{D}$, and Supplemental Figure S3B). Therefore, Cygb deficiency triggers the early DNA damage and activation of ERK/ AKT pathways, leading to the rapid progression of steatohepatitis to cancer development with CDAA treatment.

\section{Increased 0xidative Stress in Cygb Deficiency}

Next, because the CDAA diet induced oxidative stress ${ }^{16}$ and Cygb can scavenger NO and ROS produced during oxidative stress ${ }^{10}$ we hypothesized that the oxidative stress conditions induced by a CDAA diet must be more stringent in the livers of $\mathrm{Cygb}^{-1-}$ mice than in those of WT mice. Thus, we examined the level of ROS and related molecules in CDAAtreated $\mathrm{Cygb}^{-1-}$ mice at the 32-week point. DHE staining showed stronger accumulation of red fluorescence in the nuclei of hepatocytes of $C y g b^{-1-}$ mice compared to WT mice (Figure 4A). Identical phenomenon was found from the 8week point in CDAA-treated $\mathrm{Cyg}^{-1-}$ mice, but not in WT ones and CSAA diet groups (Supplemental Figure S3C). In addition, we observed the following: i) an increase in hypoxic hepatocytes as shown by pimonidazole staining, ii) an induction of NO synthase and HO-1, and iii) high levels of nitrotyrosine formation in $\mathrm{Cygh}^{-1-}$ mice (Figure 4, A and B). Taken together, these results indicate that the livers of CDAA-treated $\mathrm{Cygb}^{-1-}$ mice were under stronger oxidative stress and hypoxia compared to corresponding WT mice.

Moreover, the oxidative stress and antioxidant defense PCR array revealed dysregulation of 31 genes, including an increase in pro-oxidant Mpo (39-fold) and a decrease in antioxidant genes after a 16 -week CDAA treatment course (Table 3 and Supplemental Table S1). Increased Mpo and its main source, neutrophils, was confirmed (Figure 4C). These results are likely to correlate with the increased expression of inducible NO synthase, because myeloperoxidase is involved in $\mathrm{ONOO}^{-}$catabolism. ${ }^{26}$ Therefore, the recruitment of 
A

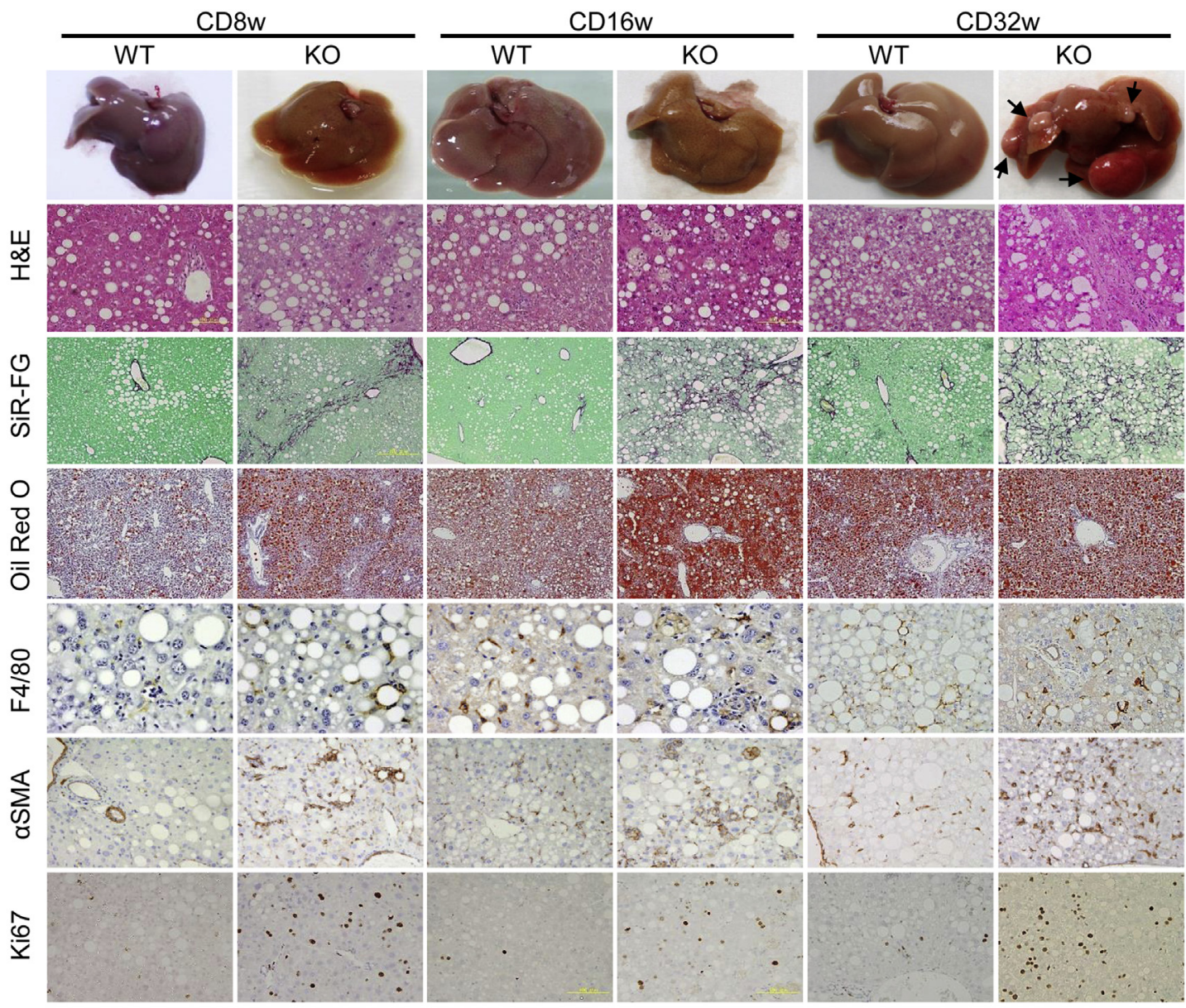

B
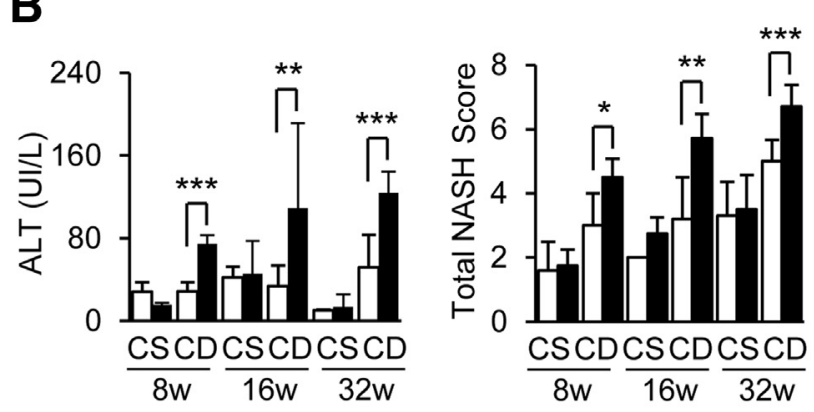

C
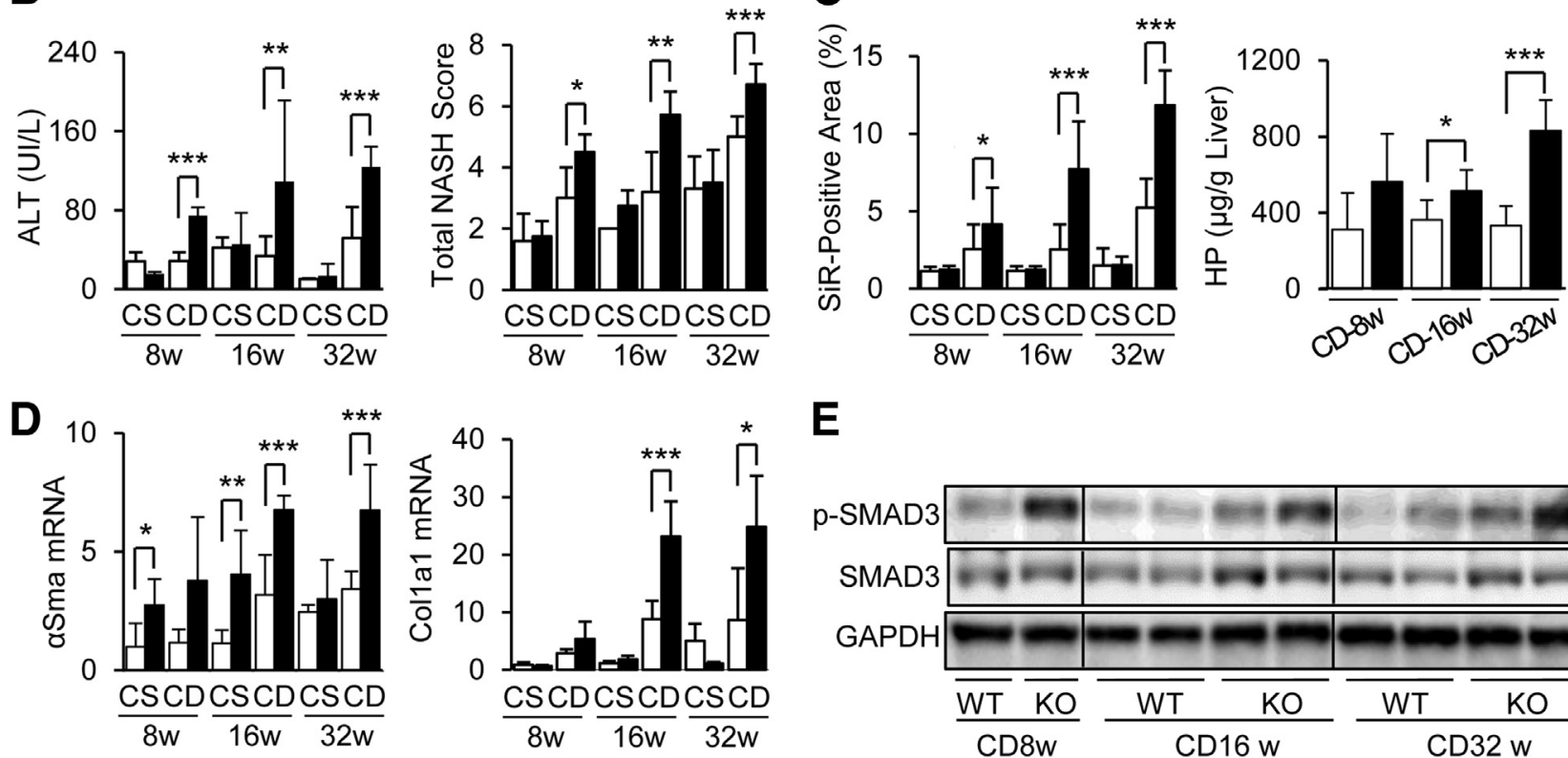

$\mathbf{E}$

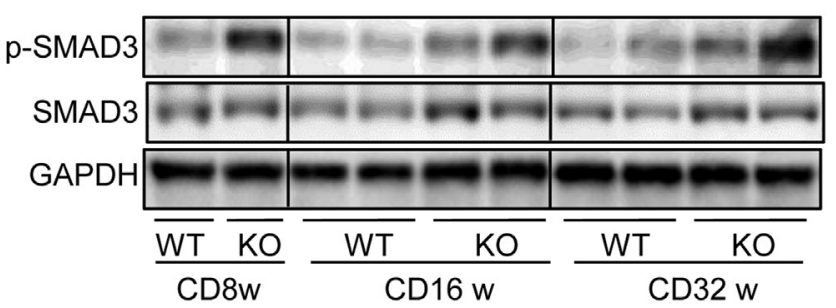

Figure 2 Promotion of hepatic steatosis and fibrosis in choline-deficient amino acid (CDAA)-fed Cygb ${ }^{-/-}$mice. Wild-type (WT) and Cygb ${ }^{-/-}$(K0) mice were fed choline-supplied amino acid (CS) or CDAA (CD) diets for 8, 16, or 32 weeks (w). A: Representative microscopic images and microscopic liver sections stained with hematoxylin and eosin (H\&E), Sirius Red and Fast Green (SiR-FG), 0il Red 0, and immunohistochemical staining for F4/80, $\alpha$-smooth muscle actin ( $\alpha$-SMA), and Ki-67. The arrows indicate tumor nodules. B: Serum alanine aminotransferase (ALT) and total nonalcoholic steatohepatitis (NASH) score. C: Sirius Red-positive area and hydroxyproline (HP) content of the liver. D: Hepatic levels of $\alpha$-Sma and collagen (Col) 1a1 mRNA. E: Immunoblots for phospho- and total SMAD3. Glyceraldehyde-3-phosphate dehydrogenase (GAPDH) is the loading control. White bar, WT; black bar, Cygb $b^{-/-}$. Data represent the means \pm SD. $n=5$ to 14 per group. ${ }^{*} P<0.05,{ }^{* *} P<0.01$, and ${ }^{* *} P<0$ 0.001. Original magnifications: $\times 200$ (SiR-FG and 0il Red 0; A); $\times 400$ (H\&E, F4/80, $\alpha-$ SMA, and Ki-67; A). 
A

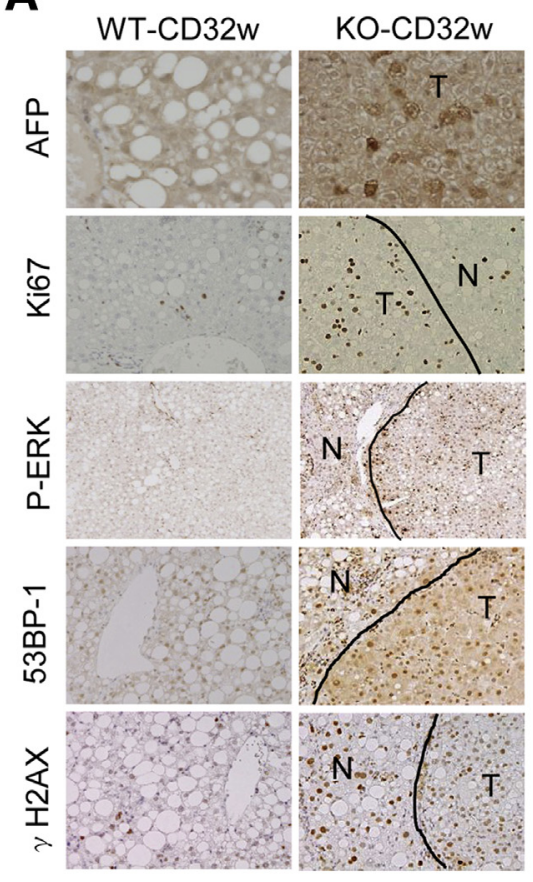

C

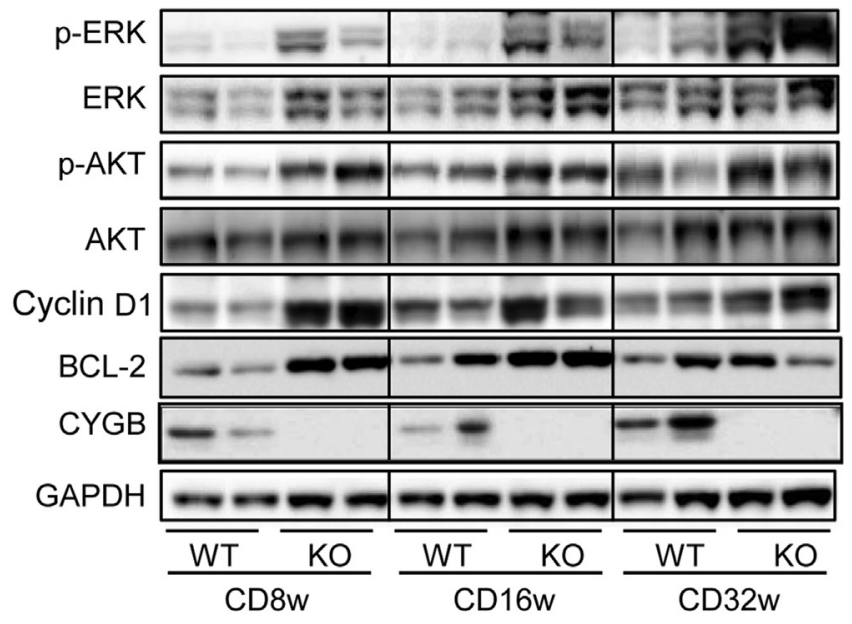

Afp

$\operatorname{Tnf} \alpha$

Tgf- $\beta 1$

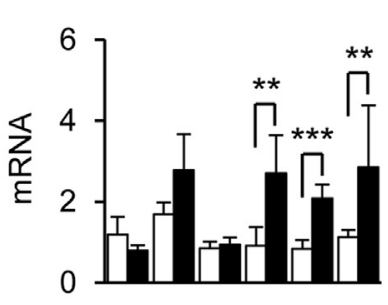

Ccl-2

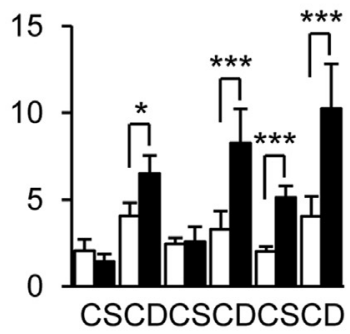

Ccl-3

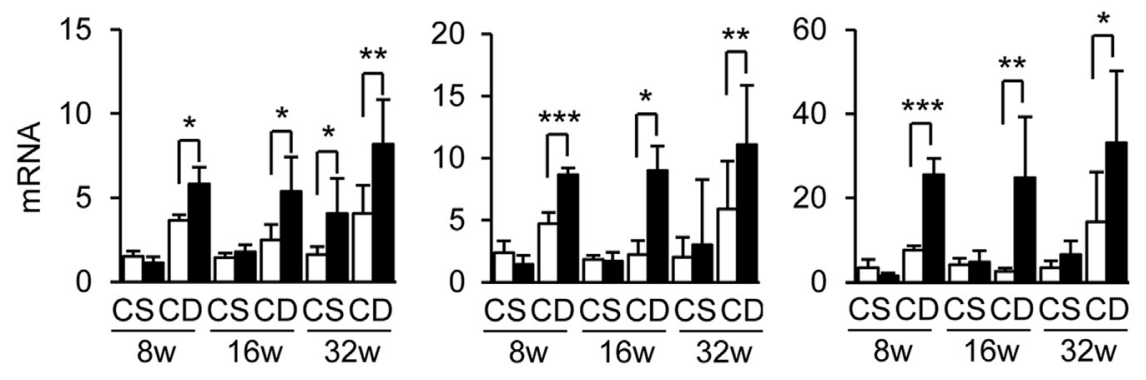

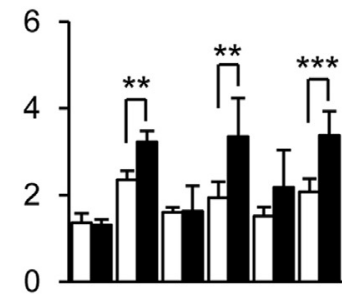

Ccl-4

D
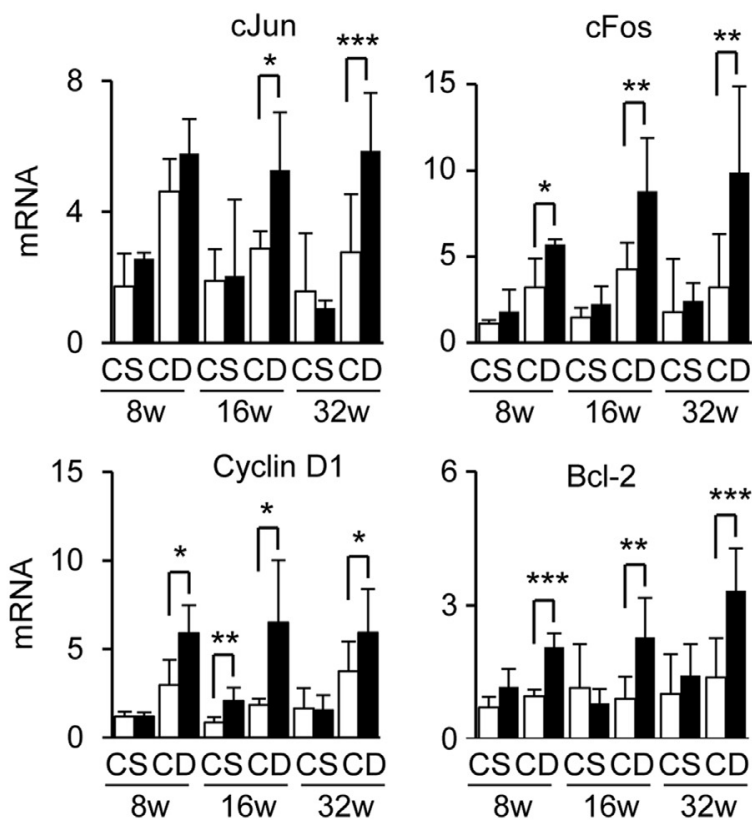

Figure 3 Augmentation of inflammation and liver cancer development in cytoglobin (CYGB) deficiency. A: Liver sections of 32-week choline-deficient amino acid (CDAA)-fed wild-type (WT) and $\mathrm{Cyg}^{-/-}(\mathrm{KO})$ mice were immunostained for $\alpha$-fetoprotein (AFP), Ki-67, p-extracellular signal-regulated kinase (ERK), 53PB-1, and phosphorylated H2A histone protein, member X ( $\gamma \mathrm{H} 2 \mathrm{AX})$. B and C: Nontumorous (N) liver tissues from WT (white bars) and Cygb ${ }^{-/-}$ (black bars) mice from Figure 2 were examined for hepatic mRNA levels of Afp, cytokines, and chemokines (B) and immunoblotted for phospho- and total ERK and AKT, CYCLIN D1, BCL-2, and CYGB (C). Glyceraldehyde-3-phosphate dehydrogenase (GAPDH) is the loading control. D: Hepatic mRNA levels of cJun, CFos, Cyclin D1, and Bcl-2. Data represent the means \pm SD. ${ }^{*} P<0.05,{ }^{*} P<0.01$, and ${ }^{* * *} P<0.001$. Original magnification, $\times 400(A)$. Ccl, chemokine ligand; $\mathrm{T}$, tumor area; Tgf, transforming growth factor; Tnf, tumor necrosis factor.

neutrophils into the liver is suspected to participate in the augmentation of oxidative stress in the $C y g b^{-1-}$ mouse liver. In contrast, glutathione peroxidase 6 and catalase-1, enzymes that degrade $\mathrm{H}_{2} \mathrm{O}_{2}$, were down-regulated in $\mathrm{Cygb}^{-1-}$ mice liver (Figure 4D) at the mRNA level.
Next, we examined whether HSCs themselves become imbalanced in terms of antioxidant/pro-oxidant levels in the absence of Cygb and generate excessive amounts of ROS and reactive nitrogen species in $\mathrm{Cygb}^{-1-}$ mice. To test this, HSCs were isolated from the livers of WT and $\mathrm{Cygb}^{-1-}$ mice 


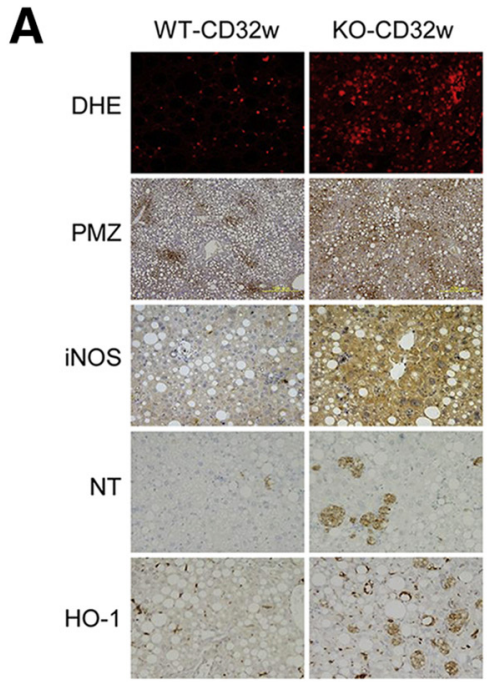

B
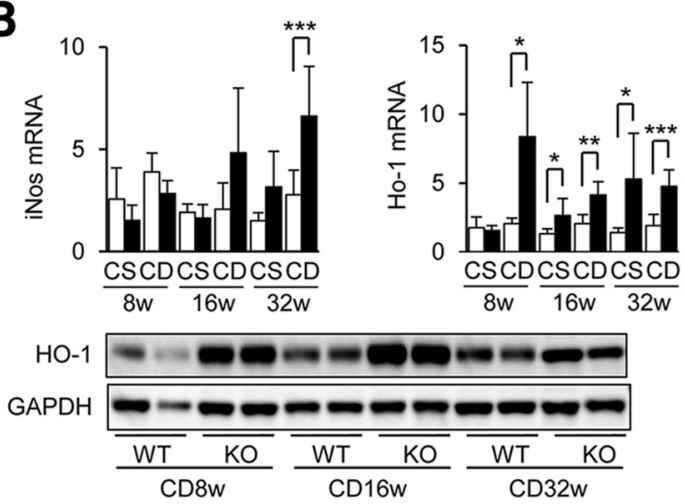

D
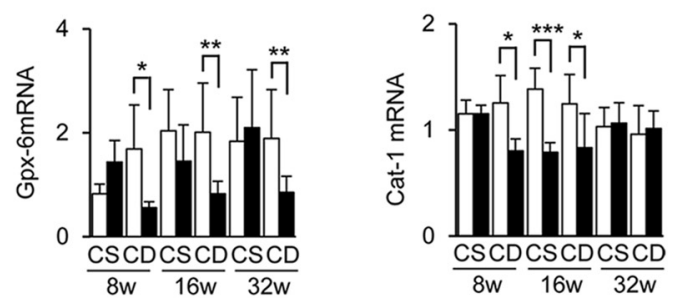

(hereafter designated $\mathrm{HSCs}^{\text {Cygb-wild }}$ and $\mathrm{HSCs}^{\text {Cygb-null, }}$ respectively) and stained for DHE (Figure 4E). It is clear that HSCs ${ }^{\text {Cygb-null }}$ showed robust fluorescent products compared to $\mathrm{HSCs}^{\text {Cygb-wild }}$ at any time point. Therefore, Cygb deficiency induced oxidative stress in HSCs in combination with the entire liver of mice fed the CDAA diet, resulting in irreversible liver injury.

\section{Blunting Inflammation, Fibrosis, and Tumor}

Development Caused by Macrophage Depletion and $\mathrm{N}$-Acetyl Cysteine Administration in Cyg ${ }^{-/-}$Mice

It is already known that activated HSCs attract and stimulate macrophages with multiple chemokines and macrophage colony-stimulating factor, and macrophages produce

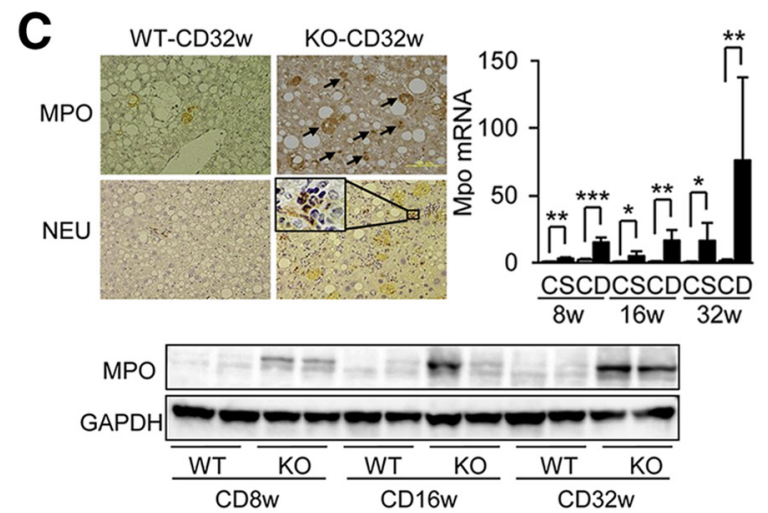

E
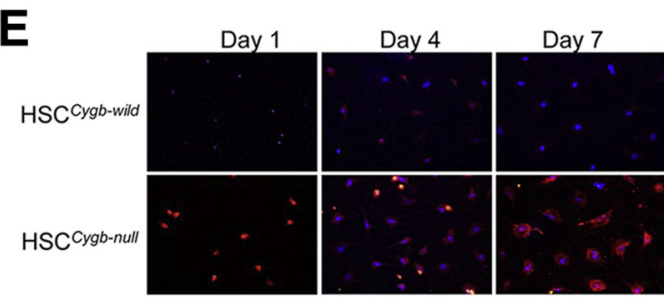

profibrotic mediators that directly activate fibroblasts. ${ }^{27}$ To gain insight into the counteraction between activated HSCs and macrophages at the onset of steatohepatitis, mice fed CDAA for 8 weeks were subjected to macrophage depletion (Supplemental Figure S4). As a result, all of the features of NASH were attenuated significantly in WT and $\mathrm{Cygb}^{-1-}$ mice (Figure 5, A and D); decreased hepatic mRNA expression levels of cytokines and fibrogenic genes (Figure 5, B and C) and of phospho- and total ERK and HO-1 at the protein level were evident (Figure 5E). Taken together, these data suggest that the macrophages, in addition to the activated HSCs, contributed to the magnification of fibroinflammatory reaction from the early stage of steatohepatitis in $\mathrm{Cyg}^{-1-}$ mice.

Next, we assessed whether NAC, a well-known antioxidative agent, is able to ameliorate the oxidative 
Table 3 List of Oxidative Defense and Antioxidant Genes Induced or Inhibited in Cygb ${ }^{-/}$Mice Fed CDAA Diet for 16 Weeks Compared with WT

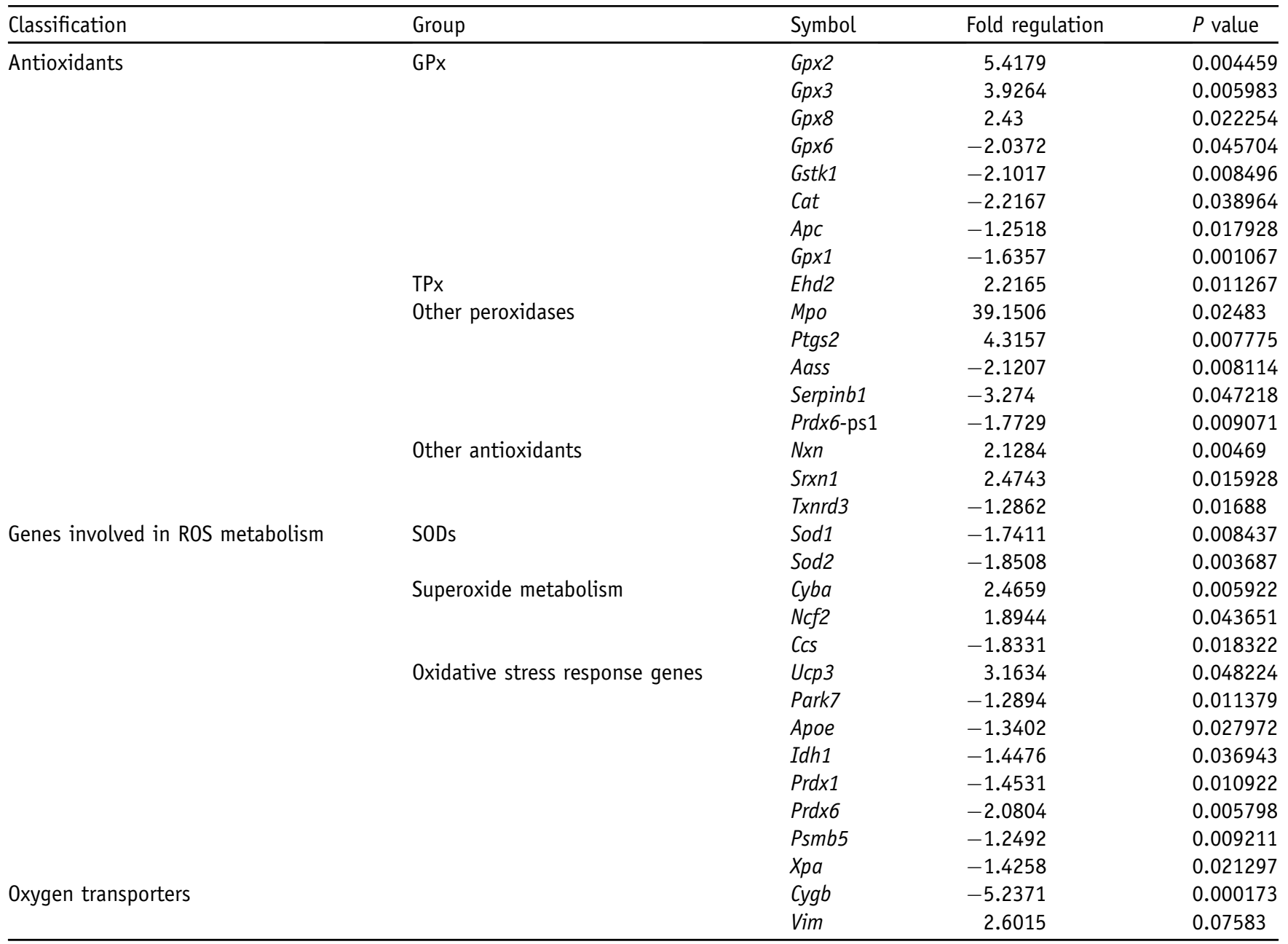

The significance of the change in gene expression between the two groups was evaluated by unpaired Student's $t$-test for each gene. The level of statistical significance is set at $P<0.05 . n=3$ for each group.

CDAA, choline-deficient amino acid; GPx, glutathione peroxidases; ROS, reactive oxygen species; SOD, superoxide dismutase; TPx, peroxiredoxins; WT, wild type.

stress-induced activation of HSCs and liver tumor formation in $C y g b^{-1-}$ mice. After CDAA feeding for 8, but not 2, weeks, $C y g b^{-1-}$ mice cotreated with $0.1 \mathrm{mmol} / \mathrm{L}$ NAC in drinking water had gained liver weight and reduced fibrosis level, as those of CDAA-treated WT mice (Supplemental Figure S5, A and B). NAC treatment also blunted the increase in $\mathrm{CD}^{+}{ }^{+}$cells (Supplemental Figure S5A) and attenuated the expression of all of the markers examined (ie, iNos, Ho-1, Tnf- $\alpha$, and $\alpha$-Sma) in CDAA-treated $\mathrm{Cyg}^{-/-}$ mice (Supplemental Figure S5, B and C).

These phenomena were found with prolonged NAC treatment for 32 weeks, with impressively reduced liver tumor formation (in terms of frequency, numbers, and sizes) compared to the non-NAC group (Figure 6, A and B). The down-regulation of oxidative stress markers (Figure 6C) subsequently induced decreases in $\alpha$-SMA, an HSC activation marker, and the Sirius Red-positive area (Figure 6, $\mathrm{A}, \mathrm{D}$, and $\mathrm{E}), \mathrm{CD}^{+} 8^{+}$cells, and inflammatory cytokines and chemokines (Figure 6, A and F); and proliferating hepatocytes, as shown by Ki-67 staining (Figure 6A). Overall, blunting oxidative stress mostly reduced HSC activation, fibrosis development, and ultimately tumor formation in CDAA-treated $\mathrm{Cyg}^{-1-}$ mice.

\section{Cygb Deficiency Triggers HSC Priming}

All of the above results indicate that the severe fibrosis and cancer development in CDAA-fed $\mathrm{Cyg}^{-1-}$ mice is related to HSC activation. We speculated that primary HSCs from Cygb $b^{-1-}$ mice possess a characteristic preactivated phenotype or priming condition that is rapidly fully activated on a CDAA diet. To test this hypothesis, purified HSCs from WT and $\mathrm{Cygb}^{-/-}$mice were subjected to phenotype analyses. Cytologically, $\mathrm{HSCs}^{C y g b-\text { null }}$ lost cellular lipid droplets more rapidly than $\mathrm{HSCs}^{C y g b-\text { wild }}$, and became enlarged with a developed $\alpha$-SMA network after 7 days in culture (Figure 7A). Interestingly, we found marked increases in the mRNA expression of fibrogenesis-related genes ( $\alpha \mathrm{Sma}$, 
A

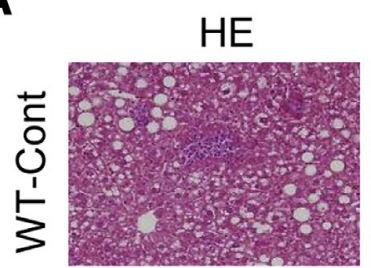

Oil Red O

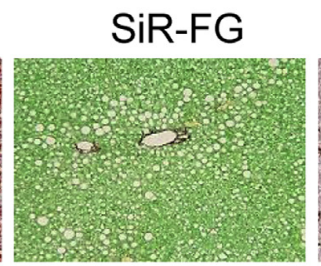

CD68

F4/80
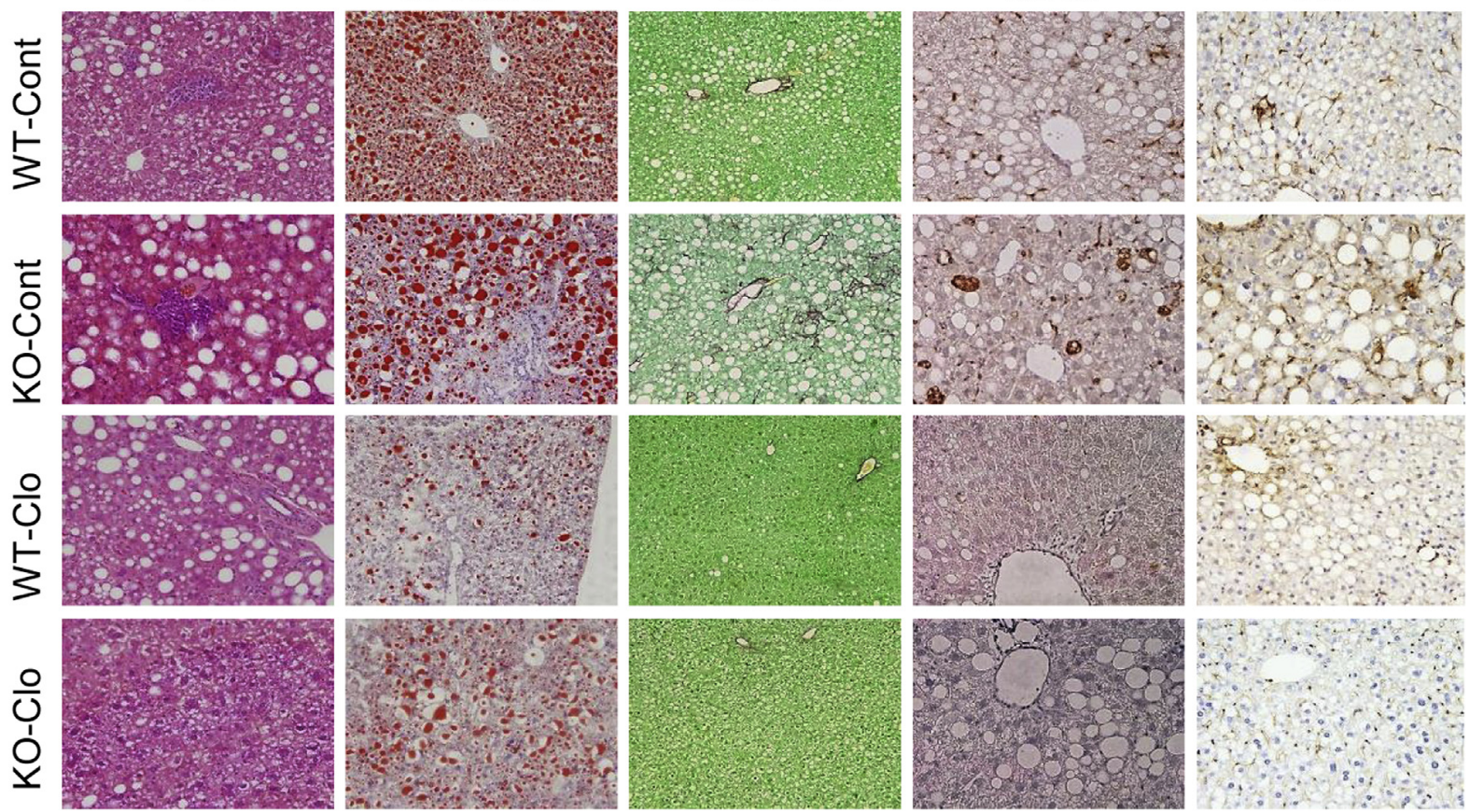

B
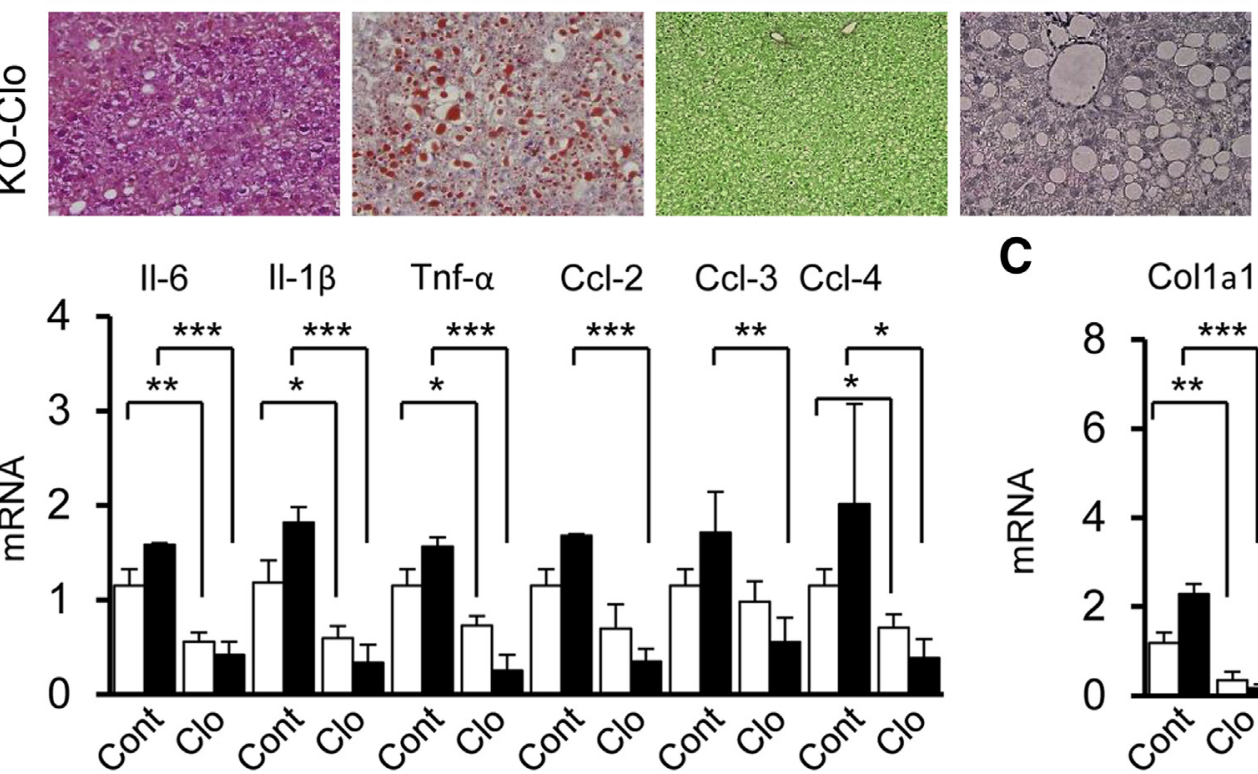

C
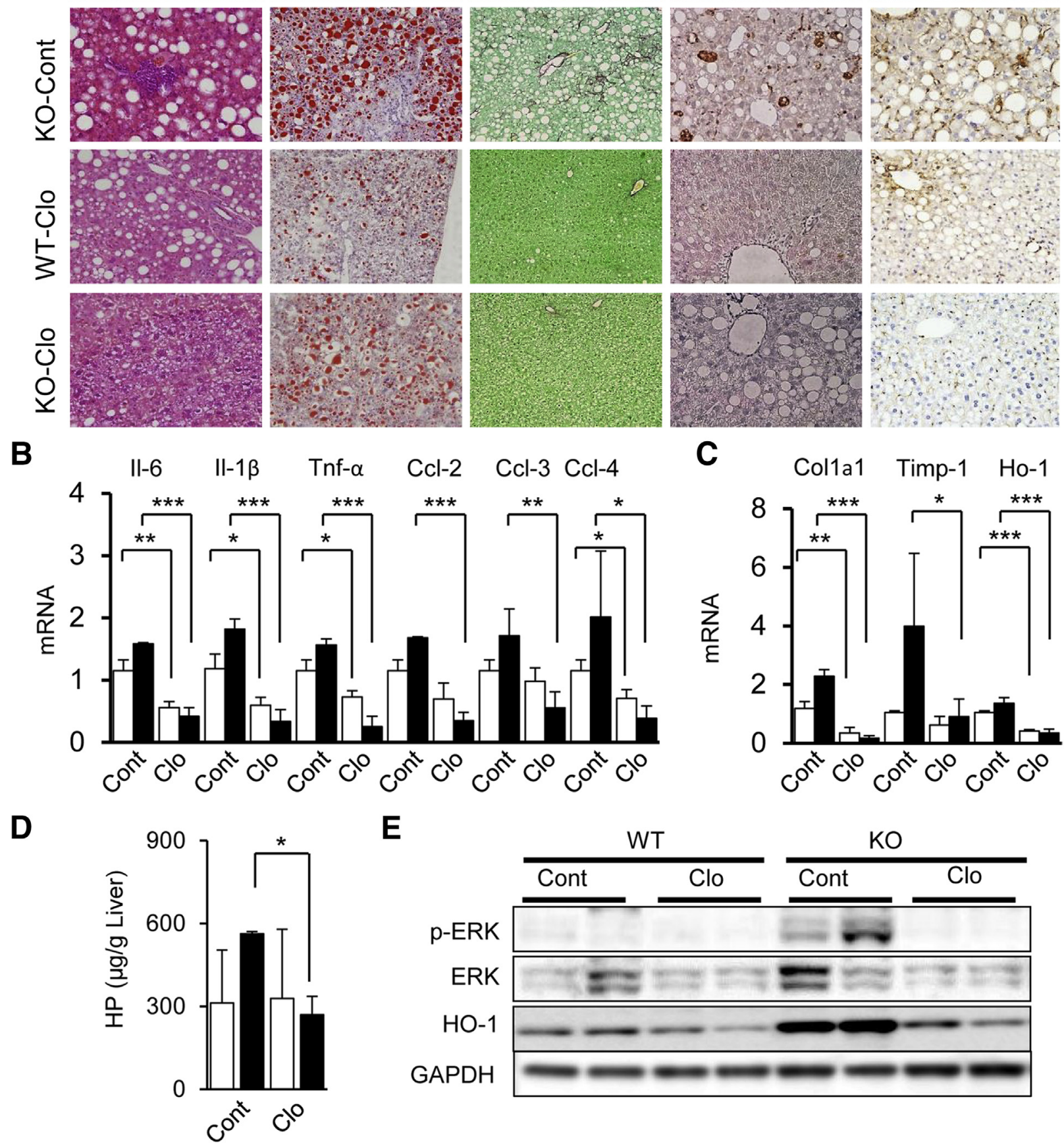

E

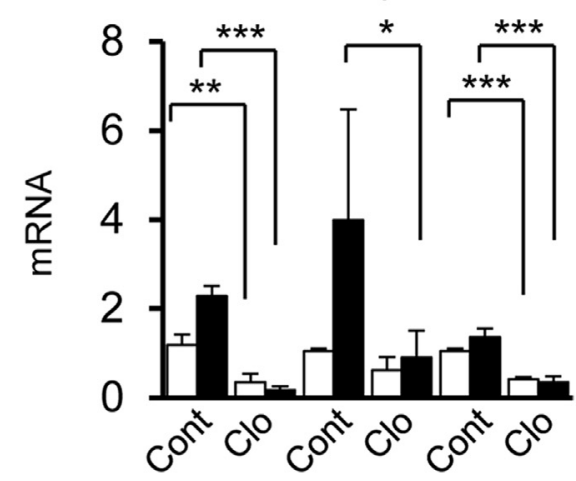

Figure 5 Effect of macrophage depletion in inflammation and fibrosis in Cygb ${ }^{-/-}$mice. Wild-type (WT; white bars) and Cygb ${ }^{-1-}$ (K0; black bars) mice were injected with liposomal clodronate (Clo) or plain control liposomes (Cont) at 7 weeks in mice fed the choline-deficient amino acid (CDAA) diet for a total of 8 weeks. A: Liver sections were stained with hematoxylin and eosin (H\&E), Oil Red 0, Sirius Red and Fast Green (SiR-FG), and immunohistochemical staining for CD68 and F4/80. B and C: Hepatic mRNA level of cytokines, chemokines (B), and fibrogenesis-related genes (C). D: Hydroxyproline (HP) content of the liver. E: Immunoblots of phospho- and total extracellular signal-regulated kinase (ERK) and heme oxygenase-1 (H0-1). Glyceraldehyde-3-phosphate dehydrogenase $\left(\right.$ GAPDH) is the loading control. Data represent the means \pm SD. $n=5$ per group. ${ }^{*} P<0.05,{ }^{*} P<0.01$, and ${ }^{* *} P<0.001$. Original magnifications: $\times 200$ (SiR-FG and 0il Red 0; A); $\times 400$ (H\&E, F4/80, and CD68; A). Ccl, chemokine ligand; Col, collagen; Timp, tissue inhibitor of metalloproteinase; Tnf, tumor necrosis factor. 


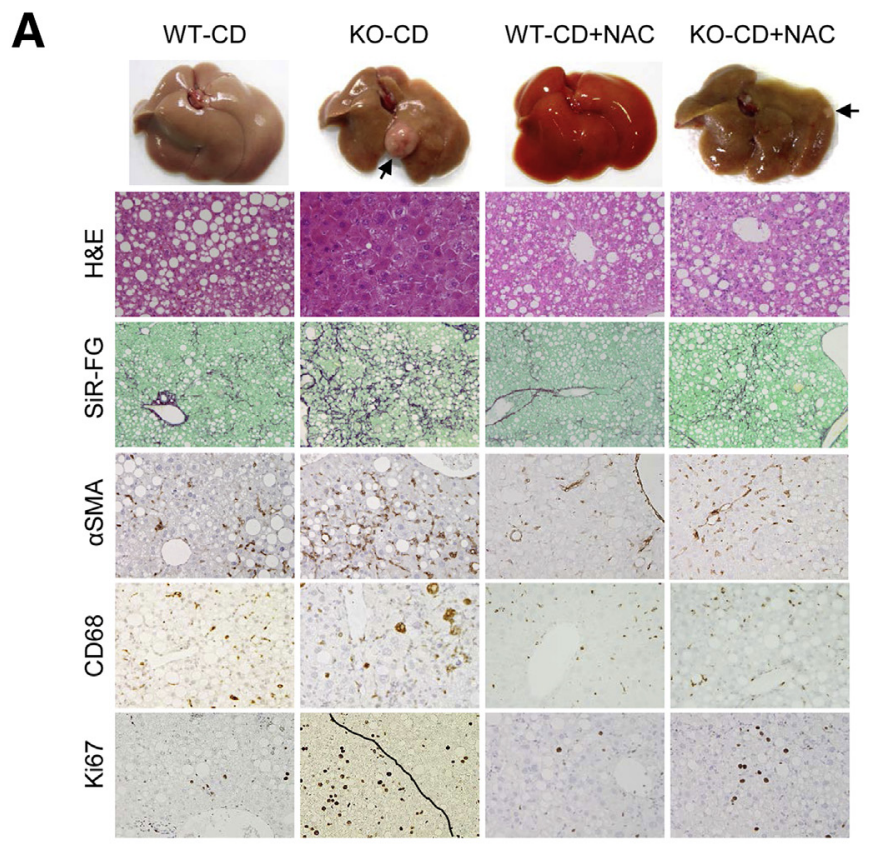

Figure 6 Inflammation, fibrosis, and tumor development ameliorate on $\mathrm{N}$-acetyl cysteine (NAC) administration in cholinedeficient (CD) amino acid-fed Cygb ${ }^{-/}$mice. Wild-type (WT) and $\mathrm{Cyg}^{-/-}$(KO) mice were fed the CDAA diet alone or in combination with NAC-treated drinking water for 32 weeks. A: Representative microscopic images and liver sections stained with hematoxylin and eosin (H\&E), Sirius Red and Fast Green (SiR-FG), and immunohistochemistry for $\alpha$-smooth muscle actin ( $\alpha$-SMA), CD68, and Ki-67, respectively. The arrow indicates a tumor nodule. B: Frequency of tumor formation, number of tumors per mouse, and maximum (Max) size of tumor in $\mathrm{Cygb}^{-1-}$ mice fed CDAA alone (KO-CD) or in combination with NAC treatment $(K O-C D+N A C)$. C $-E$ : Liver tissues from the four groups (WT-CD, KO-CD, WT-CD + NAC, and KO-CD + NAC) were analyzed to determine the levels of inducible nitric oxide synthase (iNos) and heme oxygenase-1 (Ho-1) mRNA (C), quantification of Sirius Red-positive area (D), and $\alpha$-Sma expression at the mRNA level (top panel, E). The immunoblot analysis with the glyceraldehyde-3phosphate dehydrogenase (GAPDH) loading control (bottom panel, E). F: Hepatic mRNA level of chemokine ligand (CCl)-2, Il-6, and Il-1 $\beta$. White bars indicate WT mice $(\mathbf{C}-\mathbf{F})$; black bars, Cyg $b^{-/-}$mice $(\mathbf{C}-\mathbf{F})$. Data represent the means \pm SD. $n=5$ per group. ${ }^{*} P<0.05$, ${ }^{* *} P<0.01$, and ${ }^{* * *} P<0.001$. Original magnifications: $\times 200$ (SiRFG; A); $\times 400$ (H\&E, $\alpha-S M A, C D 68$, and Ki-67; A).
B
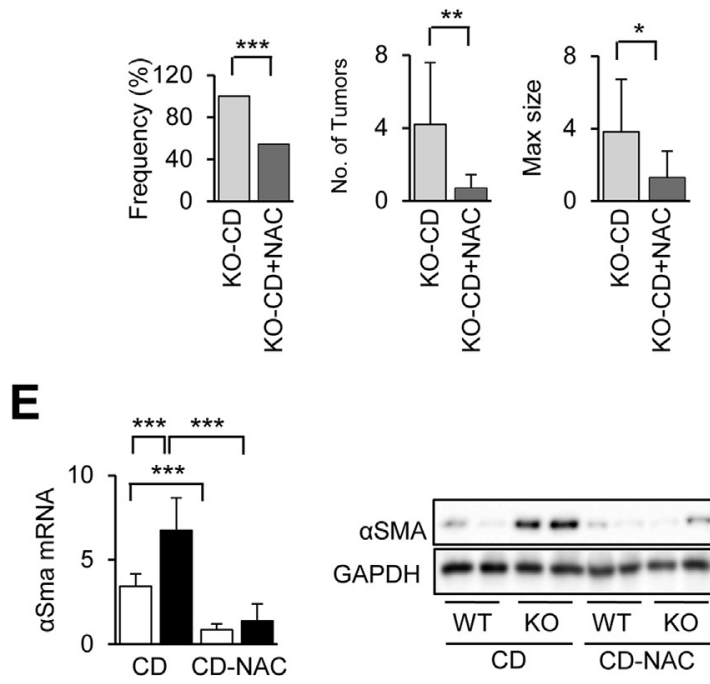

C

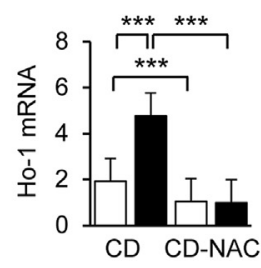

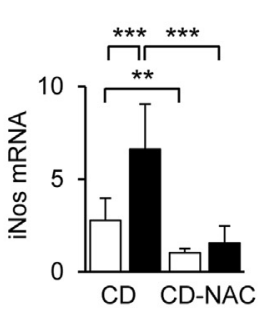

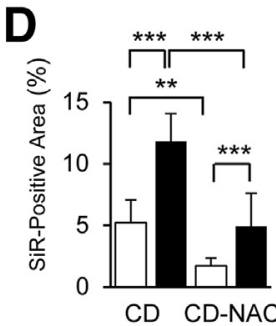

Coll $\alpha 1$, and Timp-1), cytokines (Il- 6 , Tnf- $\alpha$, and Il-1 $\beta$ ), and chemokines (Cxcls 1, 2, 5, and 7 and Ccls 2, 3, and 4) (Figure 7B) in HSCs ${ }^{\text {Cyg }}$-null at 1 day in culture, compared with $\mathrm{HSC}^{\text {Cygb-wild }}$. These differences remained until day 4, but were lost by day 7 (data not shown). Immunoblot showed an increased expression of HO-1 and p-ERK in $\mathrm{HSCs}^{\text {Cygb-null }}$ at 1 day (Figure 7C). Similar to HSCs ${ }^{\text {Cygb-null }}$,

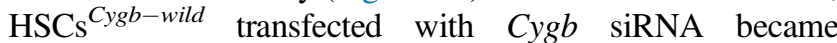
morphologically enlarged and expressed more mRNAs and proteins than the negative control (Figure 7, D and E). In contrast, HSCs ${ }^{\text {Cygb-null }}$ treated with $100 \mu \mathrm{g} / \mathrm{mL}$ of recombinant human CYGB for 72 hours showed marked reduction in $\alpha$-SMA mRNA and protein expression and maintained their quiescent morphological features (Figure 7F). Taken
$\mathbf{F}$
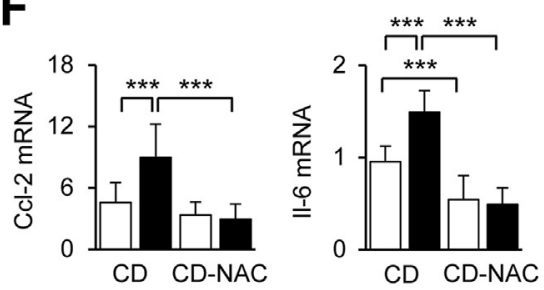

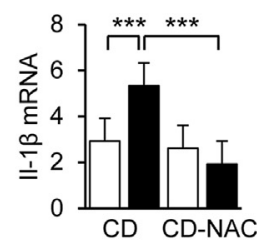

together, the loss of Cygb both in vitro and in vivo induced priming conditions in which the cells expressed high levels of fibroinflammatory genes and produced ROS.

\section{Discussion}

The current study showed that the key pathological characteristics of NASH, including fatty degeneration of hepatocytes accompanied by ROS formation, inflammation, and fibrosis, were markedly accelerated in a time-dependent manner in CDAA-fed $\mathrm{Cygb}^{-/-}$mice. In addition, the unexpected development of HCC in all of the $\mathrm{Cygb}^{-1-}$ mice is noteworthy. 
A

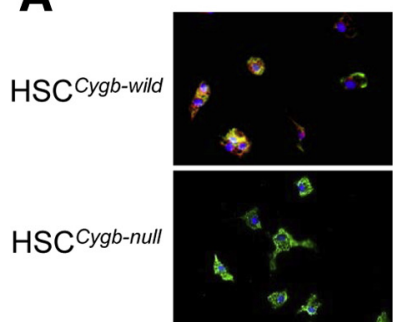

Day 1

B

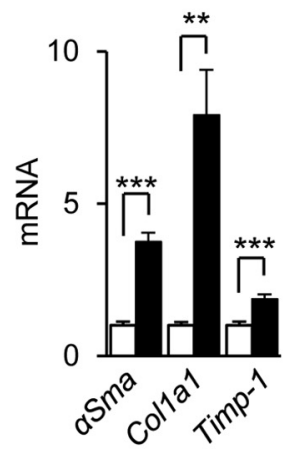

aSMA-CYGB-DAPI

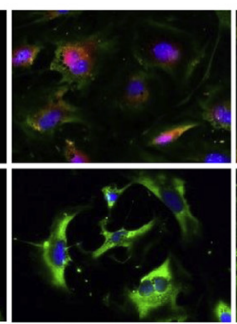

Day 4

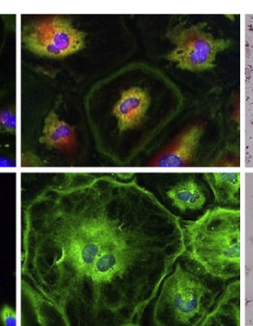

Day 7
Oil Red O

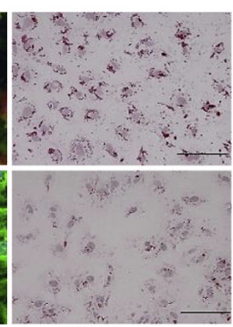

Day 7

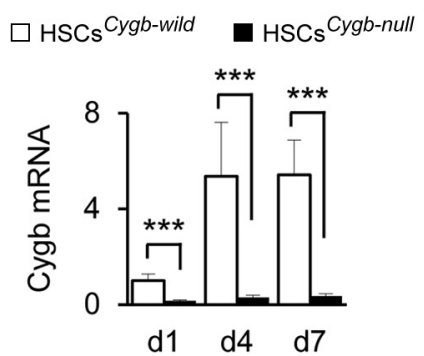

C
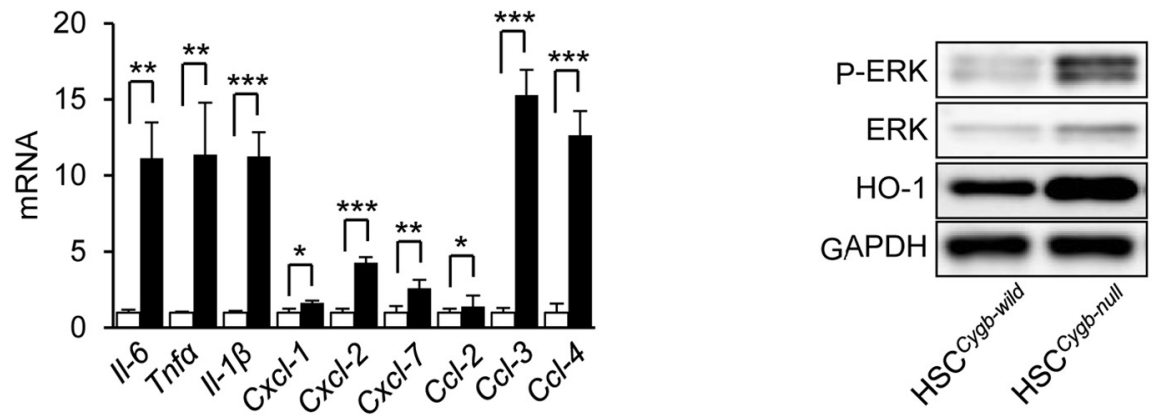

D

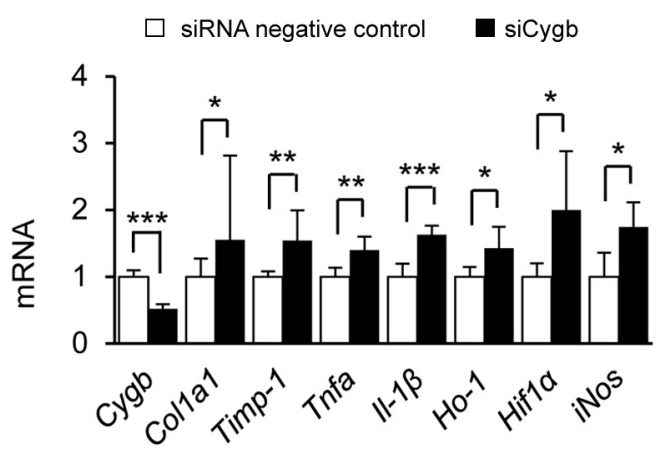

E

siNC
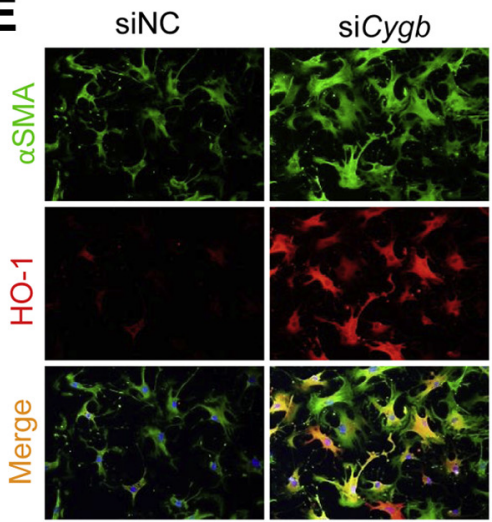

$\mathbf{F}$

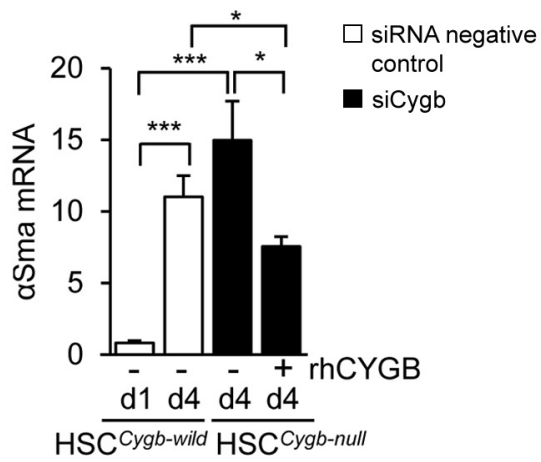

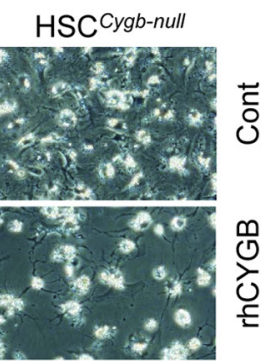

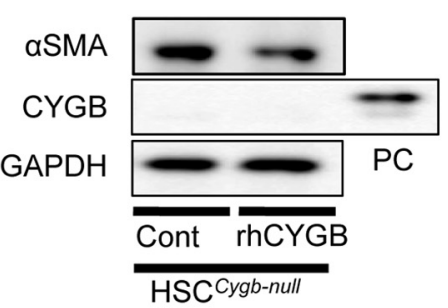

Figure 7 Priming hepatic stellate cells (HSCs) under cytoglobin (Cygb) deficiency. Primary mouse HSCs ${ }^{\text {cygb-wild }}$ and HSCs ${ }^{\text {cygb-null }}$ were cultured for days (d) 1, 4, and 7. A: Representative confocal images of $\alpha$-smooth muscle actin ( $\alpha$-SMA; green) and CYGB (red) double stain. Oil Red 0 staining was performed, and Cygb expression at the mRNA level was determined. B: mRNA expression of genes for fibrogenesis, cytokines, and chemokines at day 1. C: Immunoblots of phospho- and total extracellular signal-regulated kinase (ERK) and heme oxygenase-1 (H0-1) at day 1. Glyceraldehyde-3-phosphate dehydrogenase (GAPDH) is the loading control. D and E: Primary mouse HSCs ${ }^{\text {cygb-wild }}$ were isolated and transiently transfected with siRNA Cygb (siCygb) or siRNA-negative control for 24 hours. D: mRNA expression of fibrogenesis and oxidative stress markers. E: Representative confocal images of double staining of $\alpha$-SMA (green) and H0-1 (red). Immunoblots of CYGB, $\alpha$-SMA, H0-1, and GAPDH. F: Primary mouse HSCs ${ }^{\text {cygb-null }}$ were isolated and treated with human recombinant CYGB (rhCYGB) at the concentration of $100 \mu \mathrm{g} /$ $\mathrm{mL}$ or fresh medium (Cont) for 72 hours of $\alpha$-Sma expression at the mRNA and protein level and cell morphological features. Data represent the means \pm SD. $n=4$ to 6 per group. ${ }^{*} P<0.05,{ }^{*} P<0.01$, and ${ }^{* *} P<0.001$. Original magnification, $\times 400(\mathbf{E})$. Ccl, chemokine ligand; Col, collagen; iNos, inducible nitric oxide synthase; PC, rhCYGB serves as a positive control; Timp, tissue inhibitor of metalloproteinase; Tnf, tumor necrosis factor. 
Augmented Inflammatory Cell Infiltration with Cygb Deficiency

Increased ALT levels in $\mathrm{Cygh}^{-/-}$mice indicated more severe hepatocyte damage than that of WT mice with CDAA feeding. In addition, we found an increased number of ballooning hepatocytes that contained Mallory bodies in $\mathrm{Cygh}^{-/-}$mice (Figure 2B). The ballooned hepatocytes probably reflect imminent cell necrosis, which leads to the activation of macrophages, neutrophils, and other proinflammatory pathways. ${ }^{28}$

Infiltration of the CDAA-fed $\mathrm{Cyg}^{-/-}$mouse liver by macrophages and neutrophils was extremely pronounced from 8 weeks onward, and was accompanied by augmented cytokine and chemokine expression (Figure 3B). Ccl-2 is believed to activate HSCs and immune cells while exacerbating hepatic inflammation and cell death, contributing to the development of NASH fibrosis. ${ }^{29} \mathrm{Ccl}-3$ and $\mathrm{Ccl}-4$ trigger the recruitment of monocyte-derived macrophages and neutrophils in the liver with NASH. ${ }^{5,30}$ Thus, augmented chemokine production in Cygb deficiency promotes inflammatory cell infiltration.

\section{Aggravation of Oxidative Stress Conditions with Cygb Deficiency}

CYGB was down-regulated with human NASH and HCC, whereas the absence of $C y g b$ promotes NASH and HCC development in CDAA-treated mice. This suggests the requirement of CYGB for homeostasis in the human liver. Previous reports indicated the protective role that $C y g b$ plays in protection against oxidative stress in human neuronal cell lines ${ }^{31-33}$ and in rat HSCs. ${ }^{34}$ Our results revealed that, in addition to up-regulated pro-oxidative genes and down-regulated antioxidative genes, reactive nitrogen species accumulated in the $\mathrm{Cygb}^{-1-}$ mouse liver treated with the CDAA diet. This implies the role of $C y g b$ in $\mathrm{O}_{2}$-dependent $\mathrm{NO}$ removal as NO dioxygenase. ${ }^{35}$ Taken together, $C y g b$ plays a pivotal role in the control of ROS and reactive nitrogen species in the inflamed liver.

\section{Role of Cygb in the NASH Fibrotic Reaction}

HSCs play an important role in remodeling the extracellular matrix and the progression of fibrosis in NASH. ${ }^{12}$ Reactive oxygen intermediates, apoptotic bodies from hepatocytes, and paracrine stimuli from Kupffer cells trigger HSC activation. ${ }^{36}$ We found that loss of $C y g b$ also induced the priming of HSCs, which amplified the expression of fibrogenesis-related genes, cytokines, and a variety of chemokines (Figure 7). The priming of HSCs probably contributes to the immediate progression of fibrosis in $\mathrm{Cygh}^{-1-}$ mice. In contrast, $C y g b$ transgenic rats exhibited slow progression of fibrosis with an ischemia-reperfusion kidney injury. ${ }^{37}$ Therefore, the antifibrotic function of CYGB could be illuminative.
With regard to CYGB expression and HSC activation, we reported stellate cell activation-associated protein (original name of CYGB) and its increased expression in rat HSCs during primary culture, and in those isolated from fibrotic rat livers compared to those from normal rat livers. ${ }^{6}$ Herein, we additionally found the up-regulation of CYGB on primary-cultured mouse wild-type HSCs in vitro until day 7 and absence of CYGB in knockouts augmented HSC activation (Figure 7). In patients with $\mathrm{NASH}$, the more fibrosis developed, the less CYGB expressed in HSCs (Figure 1B). Taken together, these phenomena indicate that CYGB may be transiently induced at the early stage of HSC activation and decelerate their activation process, although the exact role of CYGB in the early stage of HSC activation should be studied further.

\section{Role of Cygb in Cancer Development with NASH}

The role of $C y g b$ as a tumor-suppressor gene has been reported in several human cancerous tissues and cancer cell lines. McRonald et $\mathrm{al}^{12}$ first reported that CYGB expression was down-regulated in tylotic esophageal biopsy specimens. Several reports have examined the decreased expression of CYGB and the hypermethylation of the $C Y G B$ promoter in non-small cell lung carcinoma tissues and head and neck cancer, among others. ${ }^{11-14,38}$ Shivapurkar et $\mathrm{al}^{39}$ reported the augmented growth of NCI-H661 lung cancer cells with siCYGB treatment, and the suppression of NCI-H228 cell proliferation when transfected with CYGB cDNA. We previously reported that $C y g b$-null mice showed susceptibility to liver tumor development under diethylnitrosamine treatment. ${ }^{15}$ These reports, along with our present study, indicate the tumorsuppressor role of $C y g b$.

DNA and aberrant mutations are known to accumulate in chronically damaged liver tissue. ${ }^{40} \gamma \mathrm{H} 2 \mathrm{AX}$, an indicator of a DNA double-stranded break, was increased in many human cancers ${ }^{41}$ human preneoplastic HCC lesions, ${ }^{42}$ and inflamed cancer tissues. ${ }^{43}$ Herein, we observed the expression of $\gamma \mathrm{H} 2 \mathrm{AX}$ and 53BP-1 in nontumor tissue regions and in tumors in Cygb-null mice (Figure 3A and Supplemental Figure S3A). Furthermore, oncogenic ERK and AKT, which are constitutively phosphorylated with $\mathrm{HCC},{ }^{40}$ were activated early in our model (Figure 3C).

In summary, $C y g b$ plays an important role in liver fibrosis and carcinogenesis through the control of HSC activation and ROS formation with a CDAA diet. The antitumorigenic and antifibrosis activity of $C y g b$ is not only model specific but may also apply to human NASH and liver cancer development.

\section{Acknowledgments}

We thank Drs. Kazuo Ikeda and Masaru Enomoto for helpful discourse and Hirano Yukiko for technical assistance. 


\section{Supplemental Data}

Supplemental material for this article can be found at http://dx.doi.org/10.1016/j.ajpath.2014.12.017.

\section{References}

1. Pessayre D, Berson A, Fromenty B, Mansouri A: Mitochondria in steatohepatitis. Semin Liver Dis 2001, 21:57-69

2. Day CP, James OF: Steatohepatitis: a tale of two "hits"? Gastroenterology 1998, 114:842-845

3. Starley BQ, Calcagno CJ, Harrison SA: Nonalcoholic fatty liver disease and hepatocellular carcinoma: a weighty connection. Hepatology 2010, 51:1820-1832

4. Ramadori G, Armbrust T: Cytokines in the liver. Eur J Gastroenterol Hepatol 2001, 13:777-784

5. Schwabe RF, Seki E, Brenner DA: Toll-like receptor signaling in the liver. Gastroenterology 2006, 130:1886-1900

6. Kawada N, Kristensen DB, Asahina K, Nakatani K, Minamiyama Y, Seki S, Yoshizato K: Characterization of a stellate cell activationassociated protein (STAP) with peroxidase activity found in rat hepatic stellate cells. J Biol Chem 2001, 276:25318-25323

7. Burmester T, Ebner B, Weich B, Hankeln T: Cytoglobin: a novel globin type ubiquitously expressed in vertebrate tissues. Mol Biol Evol 2002, 19:416-421

8. Sawai H, Kawada N, Yoshizato K, Nakajima H, Aono S, Shiro Y: Characterization of the heme environmental structure of cytoglobin, a fourth globin in humans. Biochemistry 2003, 42:5133-5142

9. Nakatani K, Okuyama H, Shimahara Y, Saeki S, Kim DH, Nakajima Y, Seki S, Kawada N, Yoshizato K: Cytoglobin/STAP, its unique localization in splanchnic fibroblast-like cells and function in organ fibrogenesis. Lab Invest 2004, 84:91-101

10. Sugimoto H, Makino M, Sawai H, Kawada N, Yoshizato K, Shiro Y: Structural basis of human cytoglobin for ligand binding. J Mol Biol 2004, 339:873-885

11. Xinarianos G, McRonald FE, Risk JM, Bowers NL, Nikolaidis G, Field JK, Liloglou T: Frequent genetic and epigenetic abnormalities contribute to the deregulation of cytoglobin in non-small cell lung cancer. Hum Mol Genet 2006, 15:2038-2044

12. McRonald FE, Liloglou T, Xinarianos G, Hill L, Rowbottom L, Langan JE, Ellis A, Shaw JM, Field JK, Risk JM: Down-regulation of the cytoglobin gene, located on 17q25, in tylosis with oesophageal cancer (TOC): evidence for trans-allele repression. Hum Mol Genet 2006, 15:1271-1277

13. Presneau N, Dewar K, Forgetta V, Provencher D, Mes-Masson AM, Tonin PN: Loss of heterozygosity and transcriptome analyses of a 1.2 $\mathrm{Mb}$ candidate ovarian cancer tumor suppressor locus region at 17q25.1-q25.2. Mol Carcinog 2005, 43:141-154

14. Shaw RJ, Omar MM, Rokadiya S, Kogera FA, Lowe D, Hall GL, Woolgar JA, Homer J, Liloglou T, Field JK, Risk JM: Cytoglobin is upregulated by tumour hypoxia and silenced by promoter hypermethylation in head and neck cancer. Br J Cancer 2009, 101:139-144

15. Thuy le TT, Morita T, Yoshida K, Wakasa K, Iizuka M, Ogawa T, Mori M, Sekiya Y, Momen S, Motoyama H, Ikeda K, Yoshizato K, Kawada N: Promotion of liver and lung tumorigenesis in DEN-treated cytoglobin-deficient mice. Am J Pathol 2011, 179:1050-1060

16. Denda A, Kitayama W, Kishida H, Murata N, Tsutsumi M, Tsujiuchi T, Nakae D, Konishi Y: Development of hepatocellular adenomas and carcinomas associated with fibrosis in C57BL/6J male mice given a choline-deficient, L-amino acid-defined diet. Jpn J Cancer Res 2002, 93:125-132

17. Matteoni CA, Younossi ZM, Gramlich T, Boparai N, Liu YC, McCullough AJ: Nonalcoholic fatty liver disease: a spectrum of clinical and pathological severity. Gastroenterology 1999, 116: 1413-1419
18. Tamori A, Nishiguchi S, Kubo S, Koh N, Moriyama Y, Fujimoto S, Takeda T, Shiomi S, Hirohashi K, Kinoshita H, Otani S, Kuroki T: Possible contribution to hepatocarcinogenesis of $\mathrm{X}$ transcript of hepatitis B virus in Japanese patients with hepatitis C virus. Hepatology 1999, 29:1429-1434

19. Committee for the Update of the Guide for the Care and Use of Laboratory Animals; National Research Council: Guide for the Care and Use of Laboratory Animals. Eighth Edition. Washington, DC, National Academies Press, 2011

20. Motoyama H, Komiya T, Thuy le TT, Tamori A, Enomoto M, Morikawa H, Iwai S, Uchida-Kobayashi S, Fujii H, Hagihara A, Kawamura E, Murakami Y, Yoshizato K, Kawada N: Cytoglobin is expressed in hepatic stellate cells, but not in myofibroblasts, in normal and fibrotic human liver. Lab Invest 2014, 94:192-207

21. Kleiner DE, Brunt EM, Van Natta M, Behling C, Contos MJ, Cummings OW, Ferrell LD, Liu YC, Torbenson MS, Unalp-Arida A, Yeh M, McCullough AJ, Sanyal AJ: Design and validation of a histological scoring system for nonalcoholic fatty liver disease. Hepatology 2005, 41:1313-1321

22. Bach Kristensen D, Kawada N, Imamura K, Miyamoto Y, Tateno C, Seki S, Kuroki T, Yoshizato K: Proteome analysis of rat hepatic stellate cells. Hepatology 2000, 32:268-277

23. Sekiya Y, Ogawa T, lizuka M, Yoshizato K, Ikeda K, Kawada N: Down-regulation of cyclin E1 expression by microRNA-195 accounts for interferon-beta-induced inhibition of hepatic stellate cell proliferation. J Cell Physiol 2011, 226:2535-2542

24. Vesselinovitch SD: The sex-dependent difference in the development of liver tumors in mice administered dimethylnitrosamine. Cancer Res 1969, 29:1024-1027

25. Naugler WE, Sakurai T, Kim S, Maeda S, Kim K, Elsharkawy AM, Karin M: Gender disparity in liver cancer due to sex differences in MyD88-dependent IL-6 production. Science 2007, 317:121-124

26. Shao B, Oda MN, Oram JF, Heinecke JW: Myeloperoxidase: an oxidative pathway for generating dysfunctional high-density lipoprotein. Chem Res Toxicol 2010, 23:447-454

27. Wynn TA, Barron L: Macrophages: master regulators of inflammation and fibrosis. Semin Liver Dis 2010, 30:245-257

28. Kono H, Rock KL: How dying cells alert the immune system to danger. Nat Rev Immunol 2008, 8:279-289

29. Chiang DJ, Pritchard MT, Nagy LE: Obesity, diabetes mellitus, and liver fibrosis. Am J Physiol Gastrointest Liver Physiol 2011, 300: G697-G702

30. Tacke F, Luedde T, Trautwein C: Inflammatory pathways in liver homeostasis and liver injury. Clin Rev Allergy Immunol 2009, 36:4-12

31. Hodges NJ, Innocent N, Dhanda S, Graham M: Cellular protection from oxidative DNA damage by over-expression of the novel globin cytoglobin in vitro. Mutagenesis 2008, 23:293-298

32. Fordel E, Thijs L, Martinet W, Lenjou M, Laufs T, Van Bockstaele D, Moens L, Dewilde S: Neuroglobin and cytoglobin overexpression protects human SH-SY5Y neuroblastoma cells against oxidative stressinduced cell death. Neurosci Lett 2006, 410:146-151

33. Fordel E, Thijs L, Martinet W, Schrijvers D, Moens L, Dewilde S: Anoxia or oxygen and glucose deprivation in SH-SY5Y cells: a step closer to the unraveling of neuroglobin and cytoglobin functions. Gene 2007, 398:114-122

34. Xu R, Harrison PM, Chen M, Li L, Tsui TY, Fung PC, Cheung PT, Wang G, Li H, Diao Y, Krissansen GW, Xu S, Farzaneh F: Cytoglobin overexpression protects against damage-induced fibrosis. Mol Ther 2006, 13:1093-1100

35. Halligan KE, Jourd'heuil FL, Jourd'heuil D: Cytoglobin is expressed in the vasculature and regulates cell respiration and proliferation via nitric oxide dioxygenation. J Biol Chem 2009, 284:8539-8547

36. Friedman SL: Mechanisms of hepatic fibrogenesis. Gastroenterology 2008, 134:1655-1669

37. Mimura I, Nangaku M, Nishi H, Inagi R, Tanaka T, Fujita T: Cytoglobin, a novel globin, plays an antifibrotic role in the kidney. Am J Physiol Renal Physiol 2010, 299:F1120-F1133 
38. Chua PJ, Yip GW, Bay BH: Cell cycle arrest induced by hydrogen peroxide is associated with modulation of oxidative stress related genes in breast cancer cells. Exp Biol Med (Maywood) 2009, 234:1086-1094

39. Shivapurkar N, Stastny V, Okumura N, Girard L, Xie Y, Prinsen C, Thunnissen FB, Wistuba II, Czerniak B, Frenkel E, Roth JA, Liloglou T, Xinarianos G, Field JK, Minna JD, Gazdar AF: Cytoglobin, the newest member of the globin family, functions as a tumor suppressor gene. Cancer Res 2008, 68:7448-7456

40. Whittaker S, Marais R, Zhu AX: The role of signaling pathways in the development and treatment of hepatocellular carcinoma. Oncogene 2010, 29:4989-5005
41. Bonner WM, Redon CE, Dickey JS, Nakamura AJ, Sedelnikova OA, Solier S, Pommier Y: $\gamma \mathrm{H} 2 \mathrm{AX}$ and cancer. Nat Rev Cancer 2008, 8: 957-967

42. Matsuda Y, Wakai T, Kubota M, Osawa M, Takamura M, Yamagiwa S, Aoyagi Y, Sanpei A, Fujimaki S: DNA damage sensor $\gamma-\mathrm{H} 2 \mathrm{AX}$ is increased in preneoplastic lesions of hepatocellular carcinoma. In The Scientific World Journal. 2013, 2013:597095. doi:10. $1155 / 2013 / 597095$

43. Murata M, Thanan R, Ma N, Kawanishi S: Role of nitrative and oxidative DNA damage in inflammation-related carcinogenesis. J Biomed Biotechnol 2012, 2012:623019 